\title{
Statistical mechanics and phase diagrams of rotating self-gravitating fermions
}

\author{
P. H. Chavanis ${ }^{1}$ and M. Rieutord ${ }^{2,3}$ \\ ${ }^{1}$ Laboratoire de Physique Théorique, Université Paul Sabatier, 118 route de Narbonne, 31062 Toulouse, France \\ 2 Observatoire Midi-Pyrénées, 14 Av. E. Belin, 31400 Toulouse, France \\ 3 Institut Universitaire de France \\ e-mail: rieutord@ast.obs-mip.fr
}

Received 3 March 2003 / Accepted 21 August 2003

\begin{abstract}
We compute statistical equilibrium states of rotating self-gravitating fermions by maximizing the Fermi-Dirac entropy at fixed mass, energy and angular momentum. We describe the phase transition from a gaseous phase to a condensed phase (corresponding to white dwarfs, neutron stars or fermion balls in dark matter models) as we vary energy and temperature. We increase the rotation up to the Keplerian limit and describe the flattening of the configuration until mass shedding occurs. At the maximum rotation, the system develops a cusp at the equator. We draw the equilibrium phase diagram of the rotating self-gravitating Fermi gas and discuss the structure of the caloric curve as a function of degeneracy parameter (or system size) and angular velocity. We argue that systems described by the Fermi-Dirac distribution in phase space do not bifurcate to non-axisymmetric structures when rotation is increased, in continuity with the case of polytropes with index $n>0.808$ (the Fermi gas at $T=0$ corresponds to $n=3 / 2$ ). This differs from the study of Votyakov et al. (2002) who consider a Fermi-Dirac distribution in configuration space appropriate to stellar formation and find "double star" structures (their model at $T=0$ corresponds to $n=0$ ). We also consider the case of classical objects described by the Boltzmann entropy and discuss the influence of rotation on the onset of gravothermal catastrophe (for globular clusters) and isothermal collapse (for molecular clouds). On general grounds, we complete previous investigations concerning the nature of phase transitions in self-gravitating systems. We emphasize the inequivalence of statistical ensembles regarding the formation of binaries (or low-mass condensates) in the microcanonical ensemble (MCE) and Dirac peaks (or massive condensates) in the canonical ensemble (CE). We also describe an hysteretic cycle between the gaseous phase and the condensed phase that are connected by a "collapse" or an "explosion".
\end{abstract}

Key words. stars: general - hydrodynamics - instabilities

\section{Introduction}

It is striking to observe that self-gravitating systems follow a kind of organization despite the diversity of their initial conditions and their environment. This organization is illustrated by morphological classification schemes such as the Hubble sequence for galaxies and by simple rules which govern the structure of individual self-gravitating systems. For example, elliptical galaxies display a quasi-universal luminosity profile described by de Vaucouleur's $R^{1 / 4}$ law and most of globular clusters are well-fitted by the Michie-King model. On the other hand, the rotation curves of spiral galaxies appear to be flat and this striking observation can be explained by the presence of a dark matter halo with a density profile decreasing as $r^{-2}$ at large distances (Binney \& Tremaine 1987).

The question that naturally emerges is what determines the particular configuration to which a self-gravitating system settles. It is possible that their actual configuration crucially

Send offprint requests to: $\mathrm{P}$. H. Chavanis,

e-mail: chavanis@irsamc.ups-tlse.fr depends on the conditions that prevail at their birth and on the details of their evolution. However, in view of their apparent regularity, it is tempting to investigate whether their organization can be favoured by some fundamental physical principles like those of thermodynamics and statistical physics. We ask therefore if the actual states of self-gravitating systems are not simply more probable than any other possible configuration, i.e. if they cannot be considered as maximum entropy states (Chavanis 2002a).

This thermodynamical approach may be particularly relevant for globular clusters and elliptical galaxies whose distribution function is close to the isothermal one (at least in the inner region of the system). In the case of globular clusters, the relaxation proceeds via two-body encounters and this "collisional" evolution is governed by the Fokker-Planck-Landau kinetic equation for which a H-theorem is available (see Kandrup 1981 for a precise discussion). This collisional evolution can lead to the establishment of a statistical equilibrium state. This equilibrium state is usually described by the Michie-King model, a truncated isothermal, which takes into account the escape of 
high energy stars due to tidal forces (Michie 1963; King 1966). In fact, such equilibria are only metastable (Antonov 1962) and the evolution continues for longer times with the formation of binaries (Hénon 1961). By contrast, for elliptical galaxies, twobody encounters are completely negligible (the corresponding relaxation time $t_{\text {coll }}$ exceeds the age of the universe by many orders of magnitude) and the galaxy dynamics is described by the Vlasov equation, i.e. collisionless Boltzmann equation. Yet, collisionless stellar systems can reach a metaequilibrium state as a result of a violent relaxation (Lynden-Bell 1967). The statistical prediction of this metaequilibrium state is complicated and depends on the initial conditions (Lynden-Bell 1967; Chavanis 2003a). Furthermore, the metaequilibrium state actually reached by the system may differ from the statistical prediction due to incomplete relaxation. Therefore, $H$-functions must be constructed to account for the structure of elliptical galaxies (Tremaine et al. 1987; Hjorth \& Madsen 1993; Chavanis 2003a,c).

The thermodynamics of self-gravitating systems started with the discoveries by Emden (1907), Bonnor (1956) and Antonov (1962) that a self-gravitating isothermal gas can be in hydrostatic equilibrium only in a limited range of thermodynamical parameters (energy, temperature and external pressure). Outside this range, the thermodynamical potential (entropy, free energy or Gibbs energy) has no extremum and the system is expected to collapse under its own gravity (Lynden-Bell \& Wood 1968). A "core-halo" phase halting the collapse can be evidenced if we introduce appropriate small-scale cut-offs. Different regularizations have been proposed such as quantum degeneracy, hard spheres, soften potential, ... Then, the shape of the caloric curve $T(E)$ depends on an additional parameter $\mu$ related to the inverse of the smallscale cut-off (see Chavanis 2002b). For $\mu \rightarrow+\infty$, one recovers the classical results of Emden, Bonnor and Antonov. As the cut-off increases, it is possible to describe phase transitions between a "gaseous" phase (independent on the smallscale cut-off) and a "condensed" phase with a core-halo structure. It is important to stress that statistical ensembles are not interchangeable for self-gravitating systems (Thirring 1970; Lynden-Bell \& Lynden-Bell 1977; Padmanabhan 1990) and that phase transitions occur not only in the canonical ensemble but also in the microcanonical ensemble if the cut-off is sufficiently small (Stahl et al. 1994; Chavanis 2002b; Chavanis \& Ispolatov 2002). Microcanonical phase transitions are a rather new phenomenon associated with the existence of metastable states (local entropy maxima).

In the previous studies, the system is supposed to be nonrotating. The thermodynamics of rotating stellar systems was studied by Lagoute \& Longaretti (1996) in the framework of Michie-King models describing globular clusters. The case of a slowly rotating isothermal gas confined within a spherical box was treated by Chavanis (2002c) using analytical methods introduced by Milne (1923) and Chandrasekhar (1933) for distorted polytropes. Recently, Fliegans \& Gross (2002) and Votyakov et al. (2002) have considered the statistical mechanics of a rapidly rotating self-gravitating gas in relation with the process of star formation. They showed that phase transitions from axisymmetric to non-axisymmetric structures ("double stars") occur at sufficiently high values of angular momentum. These authors provide an interesting phase diagram of rotating self-gravitating systems for arbitrary values of angular momentum and energy. They treat the particles as hard spheres (of size $a$ ) and consider a relatively large value of the small-scale cut-off. Fliegans \& Gross (2002) evaluate the density of state $g(E, L)$ numerically for a finite number $N$ of particles in two-dimensions. Votyakov et al. (2002) use a meanfield approximation $N \gg 1$ and adopt a distribution $\rho(\Phi)$ corresponding to a Fermi-Dirac statistics in configuration space. This model is expected to take into account hard sphere effects, where the hard spheres are introduced heuristically to fix an upper limit to the local density.

In this paper, we shall study the structure of rotating selfgravitating systems described by a Fermi-Dirac distribution function $f=f(\epsilon)$ in phase space. This is the usual distribution function of quantum particles (fermions) due to Pauli's exclusion principle. This distribution function describes electrons in white dwarf stars (Chandrasekhar 1942), neutrons in neutron stars (Hertel \& Thirring 1971) and massive neutrinos in dark matter models (Bilic \& Viollier 1997). Incidentally, this distribution function also occurs in the context of the violent relaxation of collisionless stellar systems (Chavanis \& Sommeria 1998). In the latter, the statistical approach refers to a coarsegraining of the distribution function and the effective exclusion principle is a consequence of the Liouville theorem associated with the collisionless nature of the evolution (Lynden-Bell 1967; Chavanis 2002d).

The distinction between the distribution used by Votyakov et al. (2002) and the ordinary Fermi-Dirac distribution function in phase space is crucial, regarding the structure of the condensed phase, when the system is rotating. At $T=0$ (or $E=E_{\min }$ ), the former leads to a homogeneous body ( $\rho=$ constant) while the latter leads to a polytrope with index $n=3 / 2$ corresponding to a classical white dwarf star $\left(p=K \rho^{5 / 3}\right)$. Now, it is well-known that a uniformly rotating homogeneous mass is axisymmetric (spheroidal) for slow rotations (Maclaurin sequence) but bifurcates to non-axisymmetric configurations at sufficiently high rotations (Chandrasekhar 1969). These non-axisymmetric structures are usually ellipsoidal (Jacobi sequence) but more complex structures (pearshaped, dumbbell, ...) have also been found. The fission of the self-gravitating fluid into a binary state is also possible (Hachisu \& Eriguchi 1984) and these binary states appear in the study of Votyakov et al. (2002) at low temperatures and high rotations. On general grounds, the fission instability has been extensively discussed in relation with the formation of binary stars and the formation of the moon (in the non-symmetrical case). However, the fission scenario may not be the relevant mechanism and it is almost abandoned at present. Binary stars are more likely to result from the fragmentation of the molecular cloud (Boss 1988) and the moon has probably been detached from the protoearth after a collision with a Mars-sized body (Boss 1986). In contrast to incompressible fluid masses, a polytropic configuration with index $n>0.808$ does not bifurcate to non-axisymmetric structures when angular velocity is increased; it remains axisymmetric until the Keplerian limit is reached (James 1964). At that point there is mass shedding 
at the equator (with the formation of a cusp) and disruption of the system. This concerns in particular polytropes of index $n=3 / 2$ (white dwarfs) which are the zero temperature limit of the Fermi distribution (Fowler 1926). This picture probably persists at finite temperature where we have, typically, a Fermi condensate surrounded by a "vapour". Therefore, the phase diagram corresponding to the Fermi-Dirac statistics in phase space is expected to differ from the one calculated by Votyakov et al. (2002). In this paper, we propose to compute this phase diagram for arbitrary rotation and degeneracy parameter (or system size) in both microcanonical and canonical ensembles. We shall restrict ourselves to axisymmetric singlecluster configurations because our code is limited to such configurations. According to James' result, if other structures exist (double-clusters, rings, ...), they should bifurcate discontinuously from the spheroidal sequence as rotation increases. Such bifurcations will not be considered in this paper. The present study completes the description of non-rotating or slowly rotating fermionic structures discussed by Chavanis $(2002 b, c)$.

\section{Statistical mechanics of rotating self-gravitating systems}

\subsection{The maximum entropy state}

Consider a system of $N$ particles, each of mass $m$, interacting via Newtonian gravity. We allow the system to have a non-vanishing angular momentum. Let $f(\boldsymbol{r}, \boldsymbol{v}, t)$ denote the distribution function of the system, i.e. $f(\boldsymbol{r}, \boldsymbol{v}, t) \mathrm{d}^{3} \boldsymbol{r} \mathrm{d}^{3} \boldsymbol{v}$ gives the mass of particles whose position and velocity are in the cell $\left(\boldsymbol{r}, \boldsymbol{v} ; \boldsymbol{r}+\mathrm{d}^{3} \boldsymbol{r}, \boldsymbol{v}+\mathrm{d}^{3} \boldsymbol{v}\right)$ at time $t$. The integral of $f$ over the velocity determines the spatial density

$\rho=\int f \mathrm{~d}^{3} \boldsymbol{v}$.

The mass and angular momentum of the configuration are given by

$M=\int \rho \mathrm{d}^{3} \boldsymbol{r}$,

$\boldsymbol{L}=\int f \boldsymbol{r} \times \boldsymbol{v} \mathrm{d}^{3} \boldsymbol{r} \mathrm{d}^{3} \boldsymbol{v}$.

On the other hand, in the mean-field approximation, the energy can be expressed as

$E=\frac{1}{2} \int f v^{2} \mathrm{~d}^{3} \boldsymbol{r} \mathrm{d}^{3} \boldsymbol{v}+\frac{1}{2} \int \rho \Phi \mathrm{d}^{3} \boldsymbol{r}=K+W$,

where $K$ is the kinetic energy and $W$ the potential energy. The gravitational potential $\Phi$ is related to the density by the Newton-Poisson equation

$\Delta \Phi=4 \pi G \rho$.

Let us consider different systems of astrophysical interest that can be approached by statistical mechanics. (i) The equilibrium states of collisional stellar systems (e.g., globular clusters), reached for $t>t_{\text {relax }} \sim \frac{N}{\ln N} t_{\mathrm{D}}$ (where $t_{\mathrm{D}}$ is the dynamical time), are obtained by maximizing the Boltzmann entropy (Antonov 1962; Ogorodnikov 1965; Lynden-Bell \& Wood 1968)

$S=-\int f \ln f \mathrm{~d}^{3} \boldsymbol{r} \mathrm{d}^{3} \boldsymbol{v}$

at fixed mass $M$, energy $E$ and angular momentum $\boldsymbol{L}$. This is a condition of thermodynamical stability for the Hamiltonian $\mathrm{N}$-stars system. For globular clusters, only the microcanonical ensemble makes sense and equilibrium states exist only above the Antonov energy. Furthermore, these equilibria are only metastable (but long-lived) since the Boltzmann entropy has no global maximum. (ii) Collisionless stellar systems (e.g. elliptical galaxies) can reach a metaequilibrium state on a very short time scale $\sim t_{\mathrm{D}}$ as a result of a violent relaxation. This metaequilibrium state is a stationary solution of the Vlasov equation. Lynden-Bell (1967) has tried to predict this metaequilibrium state by resorting to a new type of statistical mechanics. Unfortunately, his prediction is limited by the problem of incomplete relaxation (e.g., Hjorth \& Madsen 1993). Therefore, $\mathrm{H}$-functions must be introduced to account for the structure of galaxies (Tremaine et al. 1987; Chavanis 2003a,c). It can be shown that the maximization of a $\mathrm{H}$-function

$S=-\int C(f) \mathrm{d}^{3} \boldsymbol{r} \mathrm{d}^{3} \boldsymbol{v}$,

at fixed $M, E$ and $\boldsymbol{L}$, where $C(f)$ is a convex function (i.e. $\left.C^{\prime \prime}(f)>0\right)$ determines nonlinearly dynamically stable stationary solutions of the Vlasov equation (Ipser \& Horwitz 1979). This is similar to a condition of microcanonical stability in thermodynamics where $S$ plays the role of a generalized entropy (Chavanis 2003a). Note that according to the Jeans theorem (Binney \& Tremaine 1987), other solutions of the Vlasov equation can be constructed which do not satisfy this criterion. (iii) The equilibrium properties of stars are obtained by coupling the hydrostatic equations to a local equation of state. In simplest models, it is assumed that the star is barotropic, i.e. $p=p(\rho)$. We introduce the energy functional

$$
\begin{aligned}
\mathcal{W}[\rho]= & \int \rho \int_{0}^{\rho} \frac{p\left(\rho^{\prime}\right)}{\rho^{\prime 2}} \mathrm{~d} \rho^{\prime} \mathrm{d}^{3} \boldsymbol{r}+\frac{1}{2} \int \rho \Phi \mathrm{d}^{3} \boldsymbol{r} \\
& +\int \rho \frac{\boldsymbol{u}^{2}}{2} \mathrm{~d}^{3} \boldsymbol{r}-\boldsymbol{\Omega} \int \rho \boldsymbol{r} \times \boldsymbol{u} \mathrm{d}^{3} \boldsymbol{r},
\end{aligned}
$$

where the first term is the internal energy, the second the gravitational energy, the third the kinetic energy of the bulk motion and the fourth the energy of rotation. It can be shown that the minimization of $\mathcal{W}[\rho]$ at fixed mass $M$ and angular velocity $\boldsymbol{\Omega}$ determines nonlinearly dynamically stable stationary solutions of the Euler-Jeans equations. This is similar to a condition of canonical stability in thermodynamics where $\mathcal{W}$ plays the role of a generalized free energy (Chavanis 2003a). (iv) Finally, the interstellar medium can be considered as a gas in thermal equilibrium with a radiation background (de Vega $\&$ Sanchez 2002). Therefore, for this system, the canonical description makes sense. The stability of the gas against gravitational collapse can be studied thermodynamically by minimizing the free energy $F=E-T S$ associated with the Boltzmann entropy (6) or dynamically by using the Jeans instability criterion (see Chavanis 2002e). This is the traditional approach to understand star formation. 
In this paper, we shall consider a system of self-gravitating fermions. They can be electrons in white dwarf stars, neutrons in neutron stars or massive neutrinos in dark matter models. Their statistical equilibrium state is obtained by maximizing the Fermi-Dirac entropy

$S=-\int\left\{\frac{f}{\eta_{0}} \ln \frac{f}{\eta_{0}}+\left(1-\frac{f}{\eta_{0}}\right) \ln \left(1-\frac{f}{\eta_{0}}\right)\right\} \mathrm{d}^{3} \boldsymbol{r d}^{3} \boldsymbol{v}$,

at fixed mass, energy and angular momentum. In this formula $\eta_{0}=(2 s+1) m^{4} /(2 \pi \hbar)^{3}$ is the maximum allowable value of the distribution function fixed by Pauli's exclusion principle $(s$ is the spin). For the quantum gas, we shall consider either microcanonical or canonical equilibria. Incidentally, the FermiDirac entropy (9) also occurs in the statistical theory of violent relaxation for collisionless stellar systems developed by Lynden-Bell (1967). It corresponds to the two-levels approximation $f_{0} \in\left\{0, \eta_{0}\right\}$ of his theory, where $f_{0}$ is the initial distribution function (Chavanis \& Sommeria 1998). In the non degenerate limit $f \ll \eta_{0}$, the Fermi-Dirac entropy (9) returns the Boltzmann entropy (6) as a special case. Therefore, our study can apply either to quantum particles, stellar systems or to the interstellar medium. Of course, each system corresponds to a different limit of the theory characterized by a different degree of degeneracy and rotation.

Looking for critical points of entropy (9) at fixed mass, energy and angular momentum and introducing Lagrange multipliers $\alpha, \beta$ and $-\beta \boldsymbol{\Omega}$ for each constraint, we obtain the FermiDirac distribution function

$f=\frac{\eta_{0}}{1+\lambda \mathrm{e}^{\beta(\epsilon-\boldsymbol{\Omega} \cdot j)}}$,

where $\epsilon=\frac{v^{2}}{2}+\Phi$ and $\boldsymbol{j}=\boldsymbol{r} \times \boldsymbol{v}$ are the energy and the angular momentum of a particle by unit of mass and $\beta=1 / T$ is the inverse temperature (we include $m$ or $\eta_{0}$ in the definition of $T$ ). The same distribution (10) is obtained by extremizing the free energy $J=S-\beta E+\beta \boldsymbol{\Omega} \cdot \boldsymbol{L}$ at fixed $\beta$, $\boldsymbol{\Omega}$ and $M$. However, the second order variations of $S$ and $J$ (under respective constraints) can have a different sign implying an inequivalence of statistical ensembles (Chavanis 2003c). Introducing the Jacobi energy $\epsilon_{\mathrm{J}} \equiv \boldsymbol{\epsilon}-\boldsymbol{\Omega} \cdot \boldsymbol{j}$ and noting that $\epsilon_{\mathrm{J}}=\frac{w^{2}}{2}+\Phi_{\text {eff }}$, where $\boldsymbol{w}=\boldsymbol{v}-\boldsymbol{\Omega} \times \boldsymbol{r}$ is the relative velocity and $\Phi_{\text {eff }}=\Phi-\frac{1}{2}(\boldsymbol{\Omega} \times \boldsymbol{r})^{2}$ is the effective potential accounting for inertial forces, we can rewrite Eq. (10) in the form

$f=\frac{\eta_{0}}{1+\lambda \mathrm{e}^{\beta\left(\frac{w^{2}}{2}+\Phi_{\text {eff }}\right)}}$.

We note that the "most probable" form of rotation is a rigid rotation $\mathbf{\Omega}$. The gravitational potential $\Phi$ is defined within an arbitrary gauge constant. We shall take the ordinary convention $\Phi \rightarrow 0$ at infinity.

By integrating Eq. (11) over the velocity, we find that the density is given by

$\rho=\frac{4 \pi \sqrt{2} \eta_{0}}{\beta^{3 / 2}} I_{1 / 2}\left(\lambda \mathrm{e}^{\beta \Phi_{\text {eff }}}\right)$,

where $I_{n}$ is the Fermi integral

$I_{n}(t)=\int_{0}^{+\infty} \frac{x^{n}}{1+t \mathrm{e}^{x}} \mathrm{~d} x$.
The equilibrium configuration is then obtained by solving the Fermi-Poisson equation

$\Delta \Phi=\frac{16 \pi^{2} \sqrt{2} G \eta_{0}}{\beta^{3 / 2}} I_{1 / 2}\left(\lambda \mathrm{e}^{\beta \Phi_{\text {eff }}}\right)$,

and relating the Lagrange multipliers $\lambda, \beta$ and $\boldsymbol{\Omega}$ to the constraints $M, E$ and $\boldsymbol{L}$. We stress that, for long-range systems, the mean-field approximation is exact in a suitable thermodynamic limit (see Sect. 2.4) so that our "thermodynamical" approach based on the maximization of thermodynamical potentials is rigorous and simpler than the "statistical" approach based on functional integration (Laliena 1999; Votyakov et al. 2002). The relation between the two points of view is discussed in Chavanis (2003a).

\subsection{The box model}

It is easy to see that Eq. (14) has no physical solution. Indeed, the density decreases as $r^{-2}$ at large distances so that the total mass of the configuration is infinite in contradiction with our starting hypothesis. This means that there is no maximum entropy state in an unbounded domain. A self-gravitating system at non-zero temperature has the tendency to "evaporate". Therefore, the statistical mechanics of self-gravitating systems (classical or quantum) is essentially an out-of-equilibrium problem. However, evaporation is a slow process and, in practice, the system is found in a quasi-equilibrium state with a "core-halo" structure. The core of the system is approximately isothermal while the halo departs from isothermality. Therefore, the distribution function (10) must be modified at high energies. Some modified isothermal distributions have been introduced in the case of globular clusters (Michie 1963; King 1966) subject to the tides of a nearby object (typically a spiral galaxy) and in the case of elliptical galaxies undergoing incomplete violent relaxation (Hjorth \& Madsen 1993).

Another astrophysically plausible distribution function which takes into account Pauli's exclusion principle and tidal effects is given by (Chavanis 1998)

$f=\eta_{0} \frac{\mathrm{e}^{-\beta \epsilon}-\mathrm{e}^{-\beta \epsilon_{\mathrm{m}}}}{\lambda+\mathrm{e}^{-\beta \epsilon}}$,

for $\epsilon \leq \epsilon_{\mathrm{m}}$ and $f=0$ for $\epsilon \geq \epsilon_{\mathrm{m}}$ (where $\epsilon_{\mathrm{m}}$ is the escape energy). This distribution function can be derived from a FokkerPlanck equation appropriate to self-gravitating fermions. It can describe dark matter halos limited in extension by tidal effects (and, possibly, certain collisionless stellar systems). In the classical limit, it reduces to the Michie-King model. The distribution function (15) will be studied specifically in another paper. For the present, we shall keep the full Fermi-Dirac distribution function (11) and confine the system within a spherical box of radius $R$ so as to avoid the infinite mass problem while preserving the rotational symmetry of the system. Typically, the size of the box delimitates the physical extent of the system under consideration. Putting the system in a box is a simple and crude way of handling the infinite mass problem. This is sufficient, in a first step, if we are just interested in describing phase transitions between gaseous and "core-halo" states. If, on the other hand, we wish to make more realistic models of self-gravitating 
systems, the spatial confinement must be given further consideration.

\subsection{Asymptotic limits $T=0$ and $T \rightarrow+\infty$}

It will be convenient in the following to work with dimensionless variables defined by

$$
\begin{aligned}
& x=\frac{r}{R}, \quad n=\frac{\rho}{\left(M / R^{3}\right)}, \quad \phi=\frac{\Phi}{(G M / R)}, \\
& \Lambda=-\frac{E R}{G M^{2}}, \quad \eta=\frac{\beta G M}{R}, \quad \Omega^{\prime}=\Omega\left(\frac{R^{3}}{G M}\right)^{1 / 2}, \\
& L^{\prime}=\frac{L}{\sqrt{G M^{3} R}}, \quad \mu=\eta_{0} \sqrt{512 \pi^{4} G^{3} M R^{3}} .
\end{aligned}
$$

This is equivalent to setting $M=R=G=1$ in the dimensional equations and we shall adopt this convention in the following. Then, the degeneracy parameter is given by $\mu=16 \sqrt{2} \pi^{2} \eta_{0}$. We also define $\psi=\beta \Phi$ and $\omega=\sqrt{\beta} \Omega$. Denoting by $\psi_{0}$ the value of $\psi$ at $r=0$ and introducing the uniformizing variable $k=$ $\lambda \mathrm{e}^{\psi_{0}}$, the Fermi-Poisson Eq. (14) becomes

$\Delta \psi=\mu \sqrt{T} I_{1 / 2}\left(k \mathrm{e}^{\psi-\psi_{0}-\frac{1}{2} \omega^{2} s^{2}}\right)$,

where $s=r \sin \theta$ denotes the distance to the axis of rotation. Equation (17) must be solved with the condition $\psi \rightarrow 0$ at infinity. Before solving this equation in the general case, we first discuss important asymptotic limits.

For $k \rightarrow+\infty$, we can use the limiting form of the Fermi integral

$$
I_{n}(t) \sim \frac{1}{t} \Gamma(n+1), \quad(t \rightarrow+\infty),
$$

and we obtain the Boltzmann-Poisson equation

$\Delta \psi=\frac{\mu \sqrt{\pi T}}{2 k} \mathrm{e}^{-\psi+\psi_{0}+\frac{1}{2} \omega^{2} s^{2}}$,

for a rotating isothermal gas. This corresponds to the high temperature limit of the Fermi distribution. Indeed, for $T \gg 1$ the distribution function (11) is Maxwellian

$f=\frac{\eta_{0}}{\lambda} \mathrm{e}^{-\beta\left(\frac{\omega^{2}}{2}+\Phi_{\text {eff }}\right)}$.

Alternatively, for $k \rightarrow 0$, we can use the limiting form of the Fermi integral

$I_{n}(t) \sim \frac{(-\ln t)^{n+1}}{n+1}, \quad(t \rightarrow 0)$,

and obtain the Lane-Emden equation

$\Delta \psi=\frac{2}{3} \mu \sqrt{T}\left(-\ln k-\psi+\psi_{0}+\frac{1}{2} \omega^{2} s^{2}\right)^{3 / 2}$,

for a rotating polytrope of index $n=3 / 2$. This corresponds to the low temperature limit of the Fermi distribution in which the structure is completely degenerate (white dwarf). Indeed, for $T=0$, the distribution function (11) is a step function

$f=\eta_{0} H\left(\epsilon_{\mathrm{J}}-\epsilon_{\mathrm{F}}\right)$, where $\epsilon_{\mathrm{J}}$ is the Jacobi energy, $\epsilon_{\mathrm{F}}=-(1 / \beta) \ln \lambda$ is the Fermi energy and $H$ is the Heaviside function. Therefore, the FermiDirac distribution function connects continuously isothermal and polytropic distributions for high and low temperatures.

We recall that for the self-gravitating Fermi gas at $T=0$ (white dwarf), the density vanishes at a finite radius $R_{*}$. The mass-radius relation can be written (Chandrasekhar 1942)

$M R_{*}^{3}=\frac{\chi}{\eta_{0}^{2} G^{3}}$,

with $\chi \simeq 5.9723 \times 10^{-3}$. The condition $R_{*} \leq R$ is equivalent to

$\mu \geq \mu_{*} \equiv \sqrt{512 \pi^{4} \chi} \simeq 17.259$.

More generally, we have

$\mu=\mu_{*}\left(\frac{R}{R_{*}}\right)^{3 / 2}$

Therefore, the degeneracy parameter $\mu$ is related to the ratio of the system size $R$ to the radius $R_{*}$ of a white dwarf star with mass $M$. Accordingly, a small value of $\mu$ corresponds to a large "effective" cut-off (played by Pauli's exclusion principle) or, equivalently, to a small system size. Alternatively, a large value of $\mu$ corresponds to a small "effective" cut-off or a large system size.

\subsection{The thermodynamic limit}

Before going further, we shall discuss the thermodynamic limit of self-gravitating systems. In order to precisely define the thermodynamic limit, we must introduce a small-scale regularization otherwise the density of states $g(E)$ diverges (Padmanabhan 1990). The most physical regularization is to consider quantum mechanics effects. Indeed, compact objects such as white dwarf stars and neutron stars owe their stability to quantum mechanics. The thermodynamic limit corresponds to $N \rightarrow+\infty$ such that $\Lambda=-E R / G N^{2} m^{2}, \eta=$ $\beta G N m / R, \Omega^{\prime}=\Omega\left(R^{3} / G N m\right)^{1 / 2}, L^{\prime}=L / \sqrt{G N^{3} m^{3} R}$ and $\mu=$ $\left(g m^{4} / h^{3}\right) \sqrt{512 \pi^{4} G^{3} N m R^{3}}$ are kept fixed. It is natural to fix $m$, $h$ and $G$. Then, the strict thermodynamic limit is $N \rightarrow+\infty$ with

$R \sim N^{-1 / 3}, \quad E \sim N^{7 / 3}, \quad \beta \sim N^{-4 / 3}$,

$\Omega \sim N, \quad L \sim N^{4 / 3}$.

This scaling was indicated in a previous paper (Chavanis 2002b). When this limit is taken, $S \sim N, J \sim N$ and the meanfield approximation is exact (note that the usual free energy $F=E-T S=-J / \beta$ scales as $N^{7 / 3}$ ). The scaling $M R^{3} \sim 1$ corresponds to the "white dwarf" mass-radius relation (24). The fact that $R \rightarrow 0$ as $N \rightarrow+\infty$ accounts for the natural tendency of self-gravitating systems to collapse (note that when $M$ reaches Chandrasekhar's limiting mass, Newtonian mechanics loses its relevance). We shall call (27) the quantum, or white dwarf, thermodynamic limit (QTL). It is particularly relevant for compact objects forming the condensed phase.

If we now consider the classical limit $h=0$, we see that $\mu$ becomes infinite so that it disappears from the problem. Then, the classical thermodynamic limit (CTL) is $N \rightarrow+\infty$ 
with fixed $\Lambda=-E R / G N^{2} m^{2}, \eta=\beta G N m / R, \Omega^{\prime}=$ $\Omega\left(R^{3} / G N m\right)^{1 / 2}, L^{\prime}=L / \sqrt{G N^{3} m^{3} R}$. We note that there is a freedom since we have to fix a parameter in order to determine the others. If we assume $\beta \sim 1$, the classical thermodynamic limit is $N \rightarrow+\infty$ with

$$
\begin{aligned}
& R \sim N, \quad E \sim N, \quad \beta \sim 1, \\
& \Omega \sim N^{-1}, \quad L \sim N^{2} .
\end{aligned}
$$

This has been called the "dilute limit" by de Vega \& Sanchez (2002). Again, $S \sim N, J \sim N$ (and now $F \sim N$ ) so that the mean-field approximation is exact in that limit. We recall that for $h=0$, the density of state diverges due to the existence of collapsed configurations. However, for sufficiently large $T$ and $E / N$, we know that there exists longlived metastable gaseous states (local entropy maxima). For $N \gg 1$, these metastable states have a very long lifetime, increasing as $\exp (N)$, so they constitute the most relevant astrophysical structures (Katz \& Okamoto 2000; Chavanis \& Ispolatov 2002; Chavanis 2003a). Globular clusters are in such metastable states. These gaseous states are independent on the small-scale cut-off so they are described adequately by the dilute limit (28). The consideration of these metastable states may solve the problems raised by Laliena (2003). Collapsed configurations (binaries in MCE and Dirac peaks in CE), which correspond to global maxima of entropy or free energy (i.e., true equilibrium states) must be discarded "by hands" because they are reached for unphysically large times $t \rightarrow+\infty$. They can be reached for accessible times only if the temperature and the energy are sufficiently small $\left(T<T_{\mathrm{c}}\right.$ or $\left.E<E_{\mathrm{c}}\right)$ because, in that case, the gaseous phase ceases to exist and the system undergoes gravitational collapse. Clearly, the end-state of the collapse depends on the nature of the small-scale cut-off (e.g., quantum degeneracy) and we must consider the thermodynamic limit (27) if we want to describe these condensed states.

If instead of fermions we consider a hard sphere gas, the filling factor is $\mu=R / a N^{1 / 3}$ where $a$ is the hard sphere radius (see Chavanis 2002b). Then, fixing $a, m$ and $G$, the strict thermodynamic limit is $N \rightarrow+\infty$ with

$$
\begin{aligned}
& R \sim N^{1 / 3}, \quad E \sim N^{5 / 3}, \quad \beta \sim N^{-2 / 3}, \\
& \Omega \sim 1, \quad L \sim N^{5 / 3} .
\end{aligned}
$$

Finally, if we consider a soften potential with soften parameter $r_{0}$, the cut-off parameter is $\mu=R / r_{0}$ (see Chavanis \& Ispolatov 2002). Then, fixing $r_{0}, m$ and $G$, the strict thermodynamic limit is $N \rightarrow+\infty$ with

$$
\begin{aligned}
& R \sim 1, \quad E \sim N^{2}, \quad \beta \sim N^{-1}, \\
& \Omega \sim N^{1 / 2} \quad L \sim N^{3 / 2} .
\end{aligned}
$$

The dilute limit (28) is of course unchanged and it describes gaseous metastable states independent on the small-scale cutoff. Note that other combinations keeping $\Lambda, \eta, \Omega^{\prime}$ and $L^{\prime}$ fixed can be considered (e.g., $G \sim 1 / N$ for fixed $m, R, \beta, E / N, \Omega$ and $L / N$ or $m \sim 1 / N$ for fixed $G, R, \beta, E, \Omega$ and $L$ ). Of course, the limits considered above are only formal. In reality, one does not have to make $N, R, \ldots$ go to $+\infty$ or 0 . The precise values taken by these parameters are fixed by the physics of the system under consideration (molecular clouds, globular clusters, white dwarfs, neutron stars, dark matter halos, ...). Note that even for small values of $N$, the mean-field approximation is accurate so that the thermodynamic limit makes sense even if we are not in the strict, mathematical, conditions of its application.

\subsection{Integral constraints}

According to the classical work of James (1964), rotating polytropes with index $n>0.808$ do not bifurcate to nonaxisymmetric structures when rotation is increased. Instead, they develop a cusp at the equator. Since the Fermi gas at $T=0$ is equivalent to a polytrope of index $n=3 / 2$, it is not expected to bifurcate to non-axisymmetric structures (in contrast to homogeneous bodies). We expect this property to persist at nonzero temperature since, at $T \neq 0$, we have essentially a polytrope $3 / 2$ surrounded by a dilute halo. We do not exclude the possibility of discontinuous bifurcations to non-axisymmetric structures or rings. However, in this paper, we shall restrict ourselves to single-cluster axisymmetric solutions of Eq. (17).

In that case, the angular momentum can be written $\boldsymbol{L}=I \boldsymbol{\Omega}$ where $I$ is the moment of inertia

$I=2 \pi \int_{-1}^{+1} \int_{0}^{1} \rho r^{4}\left(1-\mu^{2}\right) \mathrm{d} r \mathrm{~d} \mu$,

where $\mu=\cos \theta$. The energy can be deduced from the Virial theorem (see, e.g., Chavanis 2002c)

$E=-K+\oint p r \cdot d S$

The kinetic energy $K$ incorporates a rotational contribution

$K_{\mathrm{rot}}=\frac{1}{2} \boldsymbol{L} \cdot \boldsymbol{\Omega}=\frac{1}{2} I \Omega^{2}$,

and a thermal contribution

$K_{\text {th }}=\frac{3}{2} \int p \mathrm{~d}^{3} \boldsymbol{r}$

where $p=\frac{1}{3} \int f w^{2} \mathrm{~d}^{3} w$ is the local pressure. Using Eq. (11), it can be written

$p=\frac{8 \pi \sqrt{2} \eta_{0}}{3 \beta^{5 / 2}} I_{3 / 2}\left(\lambda \mathrm{e}^{\beta \Phi_{\mathrm{eff}}}\right)$,

or, in dimensionless form,

$p=\frac{\mu}{6 \pi} T^{5 / 2} I_{3 / 2}\left(k \mathrm{e}^{\psi-\psi_{0}-\frac{1}{2} \omega^{2} s^{2}}\right)$.

Equations (12) and (35) determine the equation of state of the Fermi gas at non-zero temperature (in a parametric form). Since $p=p(\rho)$, this gas is barotropic.

\subsection{A short description of the numerical procedure}

Using the variables introduced previously, the Gauss theorem can be written as

$\oint \nabla \psi \cdot \mathrm{d} S=\frac{4 \pi}{T}$, 
where the surface integral is computed on the spherical box. Now, using the decomposition of the gravitational potential in normalized spherical harmonics, i.e.,

$\psi=\sum_{l, m} \psi_{l m}(r) Y_{l m}(\theta, \phi)$

it turns out that

$T=\frac{4 \pi}{\psi_{00}^{\prime}(1)}$.

Therefore, Eq. (17) depends only on three parameters $\mu, \Omega$ and $k$. The degeneracy parameter $\mu$ and the angular velocity $\Omega$ specify a series of equilibria parameterized by the uniformizing variable $k$. Given these three parameters, the Fermi-Poisson Eq. (17) can be solved by an iterative procedure. For that purpose, the radial components of the potential, i.e. $\psi_{l m}(r)$, are discretized on the Gauss-Lobatto grid which ensures spectral convergence. Typically, the resolution used for the following numerical results was 100 radial grid points and a decomposition on 16 spherical harmonics.

The temperature $T(\mu, \Omega, k)$, the energy $E(\mu, \Omega, k)$ and the angular momentum $L(\mu, \Omega, k)$ are determined by Eqs. (39), (32) and (31). It is then possible to compute the caloric curves $E(T)$ for given $\mu$ and $\Omega$ (or $L$ ) by eliminating $k$ from the previous relations.

\section{Caloric curves for low values of the degeneracy parameter (high cut-off/small system)}

We first describe the figures of equilibrium of the rotating selfgravitating Fermi gas for a degeneracy parameter $\mu=100$. This value is smaller than the microcanonical critical point $\mu_{\mathrm{MTP}}=$ 2670 above which a first order microcanonical phase transition appears (Chavanis 2002b). Hence, for $\mu=100$ (small system), the influence of the small-scale cut-off (played here by the exclusion principle) is strong in average and stabilizes the system with respect to the gravothermal catastrophe (Lynden-Bell \& Wood 1968).

\subsection{Non-rotating configurations}

The caloric curve of non-rotating self-gravitating fermions has been discussed by Chavanis (2002b) in both canonical and microcanonical ensembles and for arbitrary values of the degeneracy parameter $\mu$ (or system size $R$ ). This completes the work of Hertel \& Thirring (1971) who worked in canonical ensemble and considered small system sizes. For non-rotating systems, the results obtained with fermions are similar to those obtained with other small-scale regularizations, the small-scale cut-off playing the same role as the inverse of the degeneracy parameter (Stahl et al. 1994; Follana \& Laliena 2000; Chavanis \& Ispolatov 2002).

We first discuss the microcanonical ensemble corresponding to an isolated system with fixed energy. For $\mu<\mu_{\mathrm{MTP}}=$ 2670 , there exists only one extremum of entropy for each energy and it is a global entropy maximum (GEM). The caloric curve is plotted in Fig. 1 (in this figure $\mu=1000$ ). For high energies, the density is almost homogeneous (Fig. 2g).

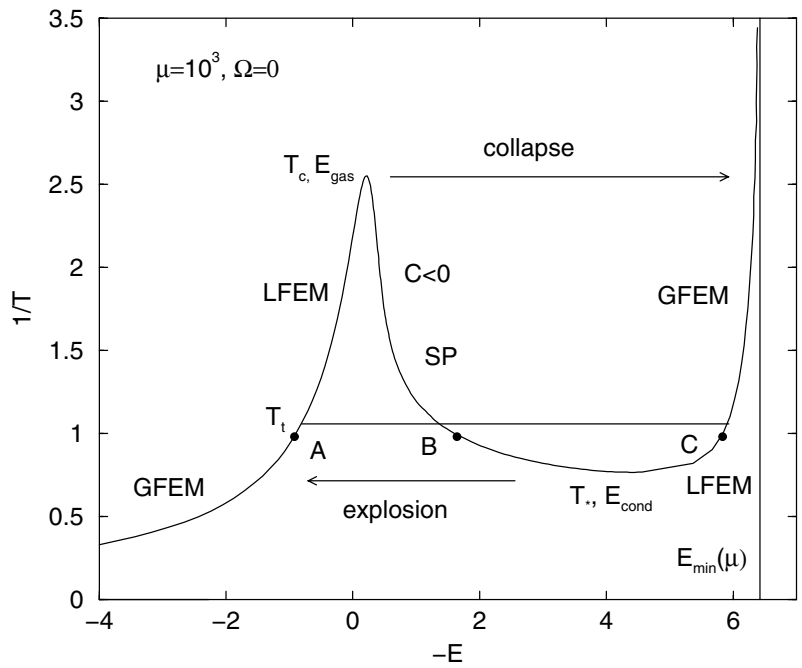

Fig. 1. The caloric curve for a non-rotating system with $\mu=1000<$ $\mu_{\mathrm{MTP}}=2670$. In the microcanonical ensemble (fixed $E$ ), the system slowly evolves from the gaseous phase to the condensed phase, passing by a mixed phase, as energy decreases. In the canonical ensemble (fixed $T$ ), the system undergoes a sharp phase transition at a critical temperature (see text). Below $T_{\mathrm{c}}$, the gaseous states undergo a gravitational collapse (Jeans instability); above $T_{*}$, the condensed states undergo an explosion. Between, $T_{\mathrm{t}}$ and $T_{\mathrm{c}}$ (resp. $T_{*}$ ) the gaseous (resp. condensed) states are metastable. By varying the temperature of the system between $T_{*}$ and $T_{\mathrm{c}}$ (spinodal points), we can generate an hysteretic cycle between the gaseous and the condensed phase.

This forms the "gaseous" phase. For energies close to the minimum accessible energy $E_{\min }=-6.42 \times 10^{-2} \mu^{2 / 3}$ (ground state), the temperature goes to zero and the system is completely degenerate (Fig. 2a). It has the same structure as a classical white dwarf star, i.e. a polytrope of index $n=3 / 2$. This forms the "condensed" phase. For intermediate energies ( $E_{\text {cond }}<E<E_{\text {gas }}$ ), corresponding to the region of negative specific heats $C=\mathrm{d} E / \mathrm{d} T<0$, the system has a "core-halo" structure with a degenerate nucleus (fermion ball) and a dilute isothermal atmosphere (Fig. 2d). This is like a solid condensate embedded in a vapour in usual phase transitions. This "mixed phase" is intermediate between the pure condensed phase and the pure gaseous phase.

We now describe the canonical ensemble in which the temperature $T$ is fixed instead of the energy $E$. Thus, we need to find maxima of free energy $J=S-\beta E$ at fixed $\beta$ and $M$. We recall that, for an isolated self-gravitating system, the validity of using the canonical ensemble to describe the statistics of a sub-system cannot be established from the microcanonical ensemble since energy is not additive (Padmanabhan 1990). Therefore, the notion of thermostat has to be defined carefully. The canonical ensemble can make sense for self-gravitating systems subject to additional short-range interactions. They can constitute a thermostat imposing a temperature. In that case, the usual canonical ensemble interpretation holds. For example, a self-gravitating system in contact with a radiation background (e.g., a molecular cloud) is usually treated in the canonical ensemble. This is the traditional way of studying stellar formation in relation with Jeans instability. Note that there exists a precise theoretical model where the canonical ensemble is 

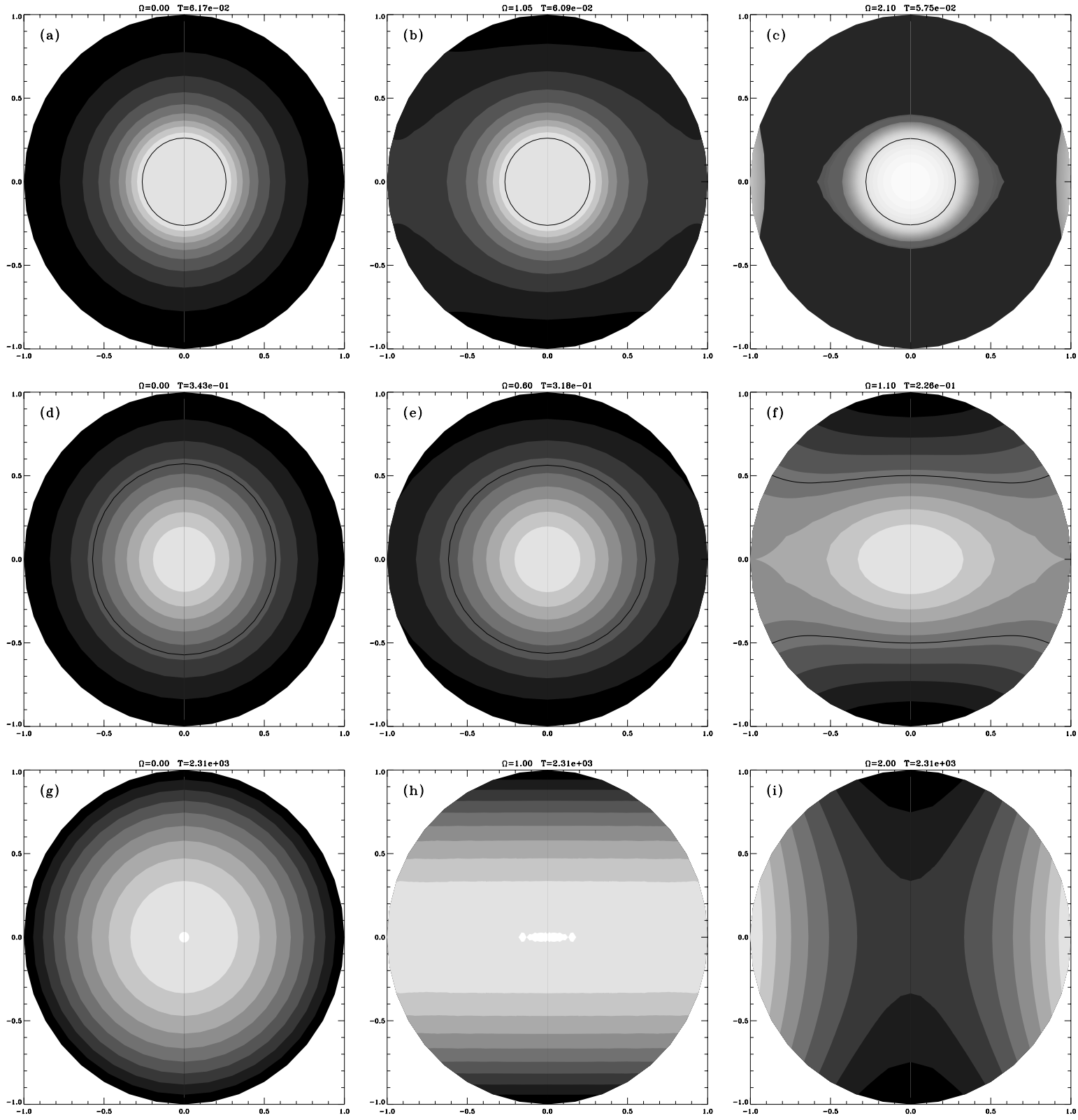

Fig. 2. Plot of the density (logarithmic scale) in a meridional section of the box. The solid line marks the level where density is 0.05 , the central density being unity. The first row shows configurations at very low temperature/energy (condensed phase). The second row shows configurations in the region of negative specific heats (mixed phase). Such structures are forbidden (unstable) in the canonical ensemble. The third row shows configurations at high temperature/energy (gaseous phase). Alternatively, the first column corresponds to non-rotating systems, the second column to slowly rotating systems and the third column to systems rotating close to the Keplerian limit.

rigorously justified. This is the self-gravitating Brownian gas model (Chavanis et al. 2002; Sire \& Chavanis 2002, 2003). In this model, the temperature has a precise physical interpretation since it is related to the strength of the stochastic force ("kicks") acting on the particles.

In the canonical ensemble, the region of negative specific heat is unstable as it corresponds to saddle points (SP) of free energy (Katz 1978; Chavanis \& Sommeria 1998). It is replaced by a canonical first order phase transition connecting the gaseous phase for $T>T_{\mathrm{t}}(\mu)$ to the condensed phase for $T<T_{\mathrm{t}}(\mu)$. Therefore, the mixed "core-halo" states with $C<0$ are thermodynamically forbidden in the canonical ensemble. The gaseous states with temperature $T_{\mathrm{c}}<T<T_{\mathrm{t}}$ are metastable (local maxima of free energy LFEM) but they are long-lived. They can play an important role reminiscent of a supercooled state of the Van der Waals gas. At $T<T_{\mathrm{c}}$ they undergo a gravitational collapse (zeroth order phase transition) coinciding with Jeans instability criterion (Chavanis 2002e). For quantum particles, the outcome of this collapse is the formation of a "fermion ball" containing almost all the mass. In the classical limit $\mu \rightarrow+\infty$, the isothermal collapse leads to a Dirac peak (see Sect. 5). The condensed states with temperature $T_{\mathrm{t}}<T<T_{*}$ are also metastable and long-lived (if they are initially prepared in such states). They will undergo an "explosion", reversed to the collapse, if they are heated above $T_{*}$. This explosion transforms the dense core into a relatively 
uniform mass distribution. By varying the temperature between $T_{*}$ and $T_{\mathrm{c}}$ we can generate an hysteretic cycle in the canonical ensemble. This hysteretic cycle was described qualitatively in Chavanis (2003b). Recently, it has been followed numerically by using a model of self-gravitating Brownian fermions (Chavanis et al. 2003). The turning points of temperature where the metastable states terminate are called (canonical) spinodal points in the langage of phase transition. The first order canonical phase transition disapears for $\mu<\mu_{\text {CTP }}=83$ (canonical critical point), i.e. for very high cut-off values. This is simply because the small-scale "repulsion" prevails over gravity. More details on phase transitions in self-gravitating systems can be found in Chavanis (2002b).

\subsection{Slowly rotating configurations}

The slowly rotating configurations of an isothermal gas (possibly degenerate) can be determined semi-analytically by using perturbative expansions of the Boltzmann-Poisson or FermiPoisson equations in terms of the dimensionless rotation parameter $v=\Omega^{2} / 2 \pi G \rho_{0}$ (Chavanis 2002c). To lowest order in the expansion, the effect of rotation is just to flatten the system and to shift the onset of instability (see Sect. 5). For $T=0$ (or $E=E_{\min }$ ), we recover the distorted polytrope of index $n=3 / 2$ studied by Milne (1923) and Chandrasekhar (1933). In the region of negative specific heats, this "rotating fermion ball" is surrounded by a gaseous halo. For high energies, the system is non degenerate. Hence, the description of the equilibrium phase diagram is very similar to that of the non-rotating Fermi gas.

\subsection{Spheroidal structures}

For higher values of rotation, the flattening increases and the system takes a spheroidal structure. For $E \rightarrow E_{\min }$, we have a rapidly rotating "fermion spheroid" (Fig. 2b). This structure is completely degenerate and highly concentrated. In particular, it is insensitive to the confining box. As energy increases further, the spheroid is only partially degenerate and extends to larger and larger distances (Fig. 2e). The rotation affects in priority the low density external region while the dense core of the configuration remains almost spherical. In the region of negative specific heats ( $E_{\text {cond }}<E<E_{\text {gas }}$ ), the "spheroid" is surrounded by a diffuse halo. This corresponds to a mixed phase. This "spheroid-halo" structure generalizes the "core-halo" structure of non-rotating systems. For even larger values of energy, the spheroid would extend outside the box so that the system becomes almost one-dimensional and depends only on the axial coordinate $z$ (Fig. $2 \mathrm{~h}$ ). Note, however, that the density contrast is extremely low $(\mathcal{R}=\rho(0) / \rho(R) \simeq 1.00022)$ so that the structure is essentially homogeneous.

\subsection{Keplerian limit}

For $E=E_{\min }$, the temperature drops to zero and the system is equivalent to a pure polytrope with index $n=3 / 2$. The structure of rapidly rotating polytropes is well-known
(James 1964). For rigid polytropes with index $n<0.808$, the system is sensitive to non-axisymmetric instabilities of various forms. Therefore, at sufficiently high rotations, the spheroidal sequence bifurcates continuously to more complicated structures (Chandrasekhar 1969). However, for $n>0.808$, nonaxisymmetric instabilities are inhibited and the series of equilibria has a different evolution. The polytrope develops a cusp at the equator at a point where the gravitational force balances the centrifugal force (Fig. 2c). This is the so-called Keplerian (or break-up) limit corresponding to a critical angular velocity $\Omega_{\max }$ such that $\Omega_{\max }^{2} R_{\mathrm{e}}^{3}=G M$, where $R_{\mathrm{e}}$ is the equatorial radius. There is no equilibrium state for $\Omega>\Omega_{\max }$ as mass is ejected from the equatorial surface by the centrifugal force (mass shedding). The tendency to expulse some mass far away may be related to the formation of a Keplerian disk above $\Omega_{\max }$. In that case, we must allow for differential rotation. Mathematically, above $\Omega_{\max }$, the maximization problem leading to Eq. (11) has no solution that are everywhere differentiable. The formation of an equatorial cusp at $\Omega=$ $\Omega_{\max }$ marks the break-up of differentiability and the complete change of structure. For $\Omega>\Omega_{\max }$, the system may resemble a "cuspy condensate" + some matter accumulating at the box (ejected by the central body). We can also wonder whether non-axisymmetric structures can form above $\Omega_{\max }$. Indeed, there exists "double-cluster" solutions for polytropes with index $n>0.808$ (Hachisu \& Eriguchi 1984) that bifurcate discontinuously from the spheroidal sequence. On general grounds, it would be of interest to compare the entropy of the single cluster to the entropy of the double cluster. This problem is left for future investigations.

For $E=E_{\min }$ and $\Omega=\Omega_{\max }$, all the mass is in the cuspy polytrope. As $E$ increases along the line of maximum rotation, the polytrope extends in size and is embedded in a disk-like halo (Fig. 2f). The maximum angular velocity $\Omega_{\max }(E)$ decreases with $E$. This is because the size of the polytrope increases so that it is more easily affected by rotation. At high energies, the polytrope is very extended and the cusp would form outside the confining box. Therefore, the structure of the system is highly affected by the wall and this makes possible to achieve arbitrary large values of angular velocity. As a result, the maximum angular velocity increases with the energy when $E$ is large. For high rotations and high energies, the matter accumulates on the wall due to the centrifugal force (Fig. 2i). The density profile is close to the law

$\rho=A \mathrm{e}^{\frac{1}{2} \beta \Omega^{2} s^{2}}$,

which corresponds to the density of a rotating gas without selfgravity. Note that the iso-density lines are axial so that a complete change of symmetry has occurred from Figs. $2 \mathrm{~h}-\mathrm{i}$.

\subsection{Equilibrium phase diagram}

The caloric curve $T(E)$ is plotted in Fig. 3 for different values of angular velocity $\Omega$. It has the typical " $N$-shape" structure previously found for non-rotating and slowly rotating systems with high cut-off. The onset of instability can be determined by a turning point criterion (Katz 1978). For $\mu=100$, 


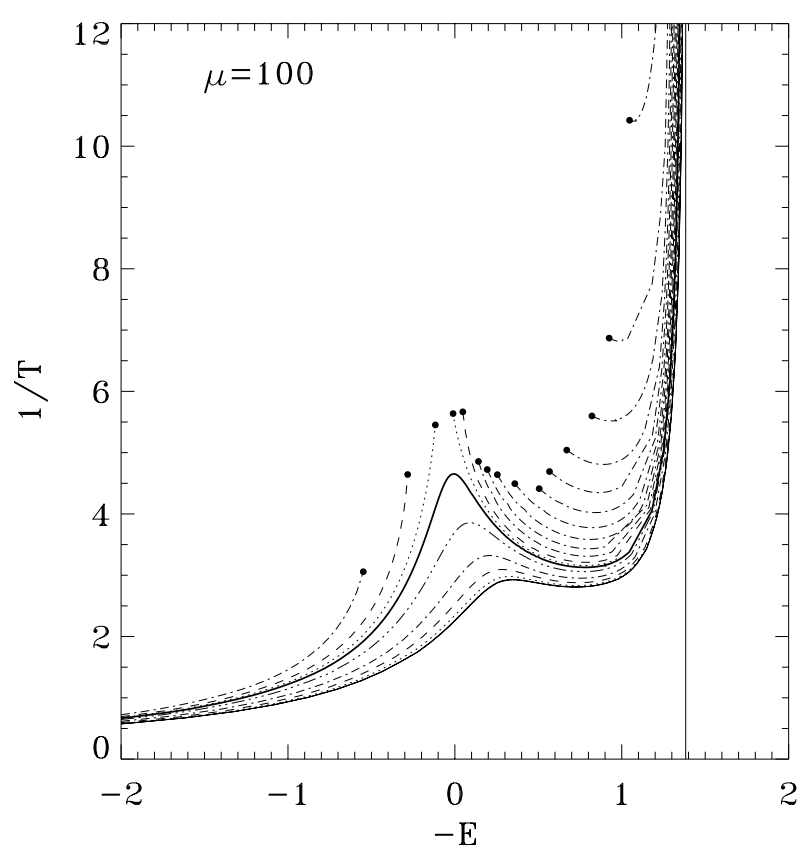

Fig. 3. The caloric curve of a rotating system with $\mu=100$. It has a $N$-shape structure characteristic of self-gravitating systems with large cut-off $\left(\mu \leq \mu_{\mathrm{MTP}}=2670\right)$. The "gaps" in energy and temperature at high rotations are due to the absence of uniformly rotating solution above the Keplerian limit. The thin full line shows the $\Omega=0$ case while the thick line marks the entry in the Kepler zone (shaded region in Figs. 4 and 5). In between lines show some intermediate cases.

the equilibrium states are always stable in the microcanonical ensemble (they are maxima of entropy at fixed mass, energy and angular momentum) but they are unstable in the canonical ensemble in the region of negative specific heats (they are saddle points of free energy at fixed mass, temperature and angular velocity). We see that, for moderate rotations, the structure of the caloric curve does not change dramatically with respect to the non-rotating case. It is just a natural continuation of previously reported results. However, at high rotations, the system is sensitive to mass shedding and there exists a maximum angular velocity $\Omega_{\max }$ for each value of energy and temperature (Keplerian limit).

The phase diagram in the $(E, \Omega)$ plane is plotted in Fig. 4. Since the energy is assumed to be fixed, this diagram corresponds to the microcanonical description ${ }^{1}$. In continuity with the non-rotating case, the horizontal structure of the diagram consists of three regions: a pure "condensed" phase for $E<$ $E_{\text {cond }}$, a pure "gaseous" phase for $E>E_{\text {gas }}$ and a mixed "corehalo" phase for intermediate energies. Vertically, the diagram proceeds with increasing flattening until the Keplerian limit is reached for $\Omega=\Omega_{\max }(E)$. Above this line, the system is

${ }^{1}$ We have used the angular velocity as a control parameter instead of the angular momentum for commodity but also because it is the most natural variable to characterize a rotating system. Indeed, the conservation of angular momentum should not be taken in a strict sense because it can always be satisfied by ejecting a small amount of mass far away with weak influence on the other constraints. For similar remarks concerning the role of angular momentum in the context of vortex dynamics, see Brands et al. (1999).

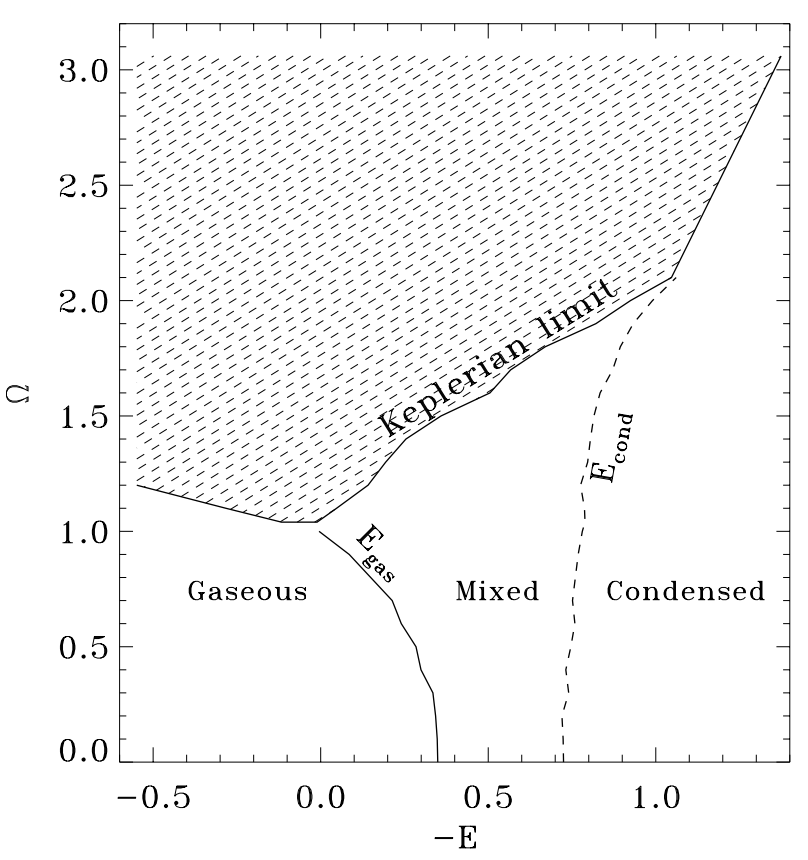

Fig. 4. Microcanonical phase diagram in the $(E, \Omega)$ plane for $\mu=100$. The maximum angular velocity $\Omega_{\max }(E)$ depends on the energy. It is maximum for the $T=0$ configuration (which corresponds to a $n=3 / 2$ polytrope). The parameters of this terminal point are $\Omega_{\max }=3.06$ and $E_{\min }=-1.37$.

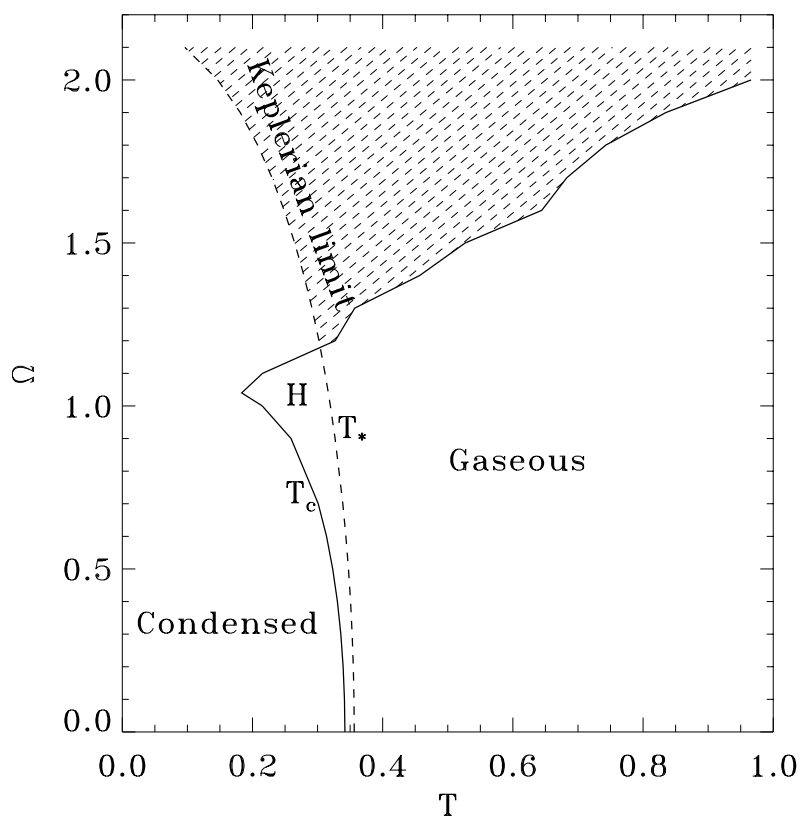

Fig. 5. Canonical phase diagram in the $(T, \Omega)$ plane for $\mu=100$. The $H$-zone corresponds to an hysteretic zone where the actual phase depends on the history of the system.

expected to expulse matter far away and, possibly, create a Keplerian disk or break into fragments.

The phase diagram in the $(T, \Omega)$ plane is plotted in Fig. 5 . Since the temperature is assumed to be fixed, this diagram corresponds to the canonical description. The mixed phase with negative specific heats allowed in the microcanonical ensemble is replaced by a phase transition connecting the "gaseous" 
phase to the "condensed" phase. Between $T_{\mathrm{c}}$ and $T_{*}$ the system can be in a metastable state. If it is initially prepared in a gaseous state, it will remain gaseous until the minimum temperature $T_{\mathrm{c}}$ at which it will collapse and become condensed. Inversely, if the system is initially prepared in a condensed state, it will remain condensed until the maximum temperature $T_{*}$ at which it will explode and become gaseous (see Fig. 1). Therefore, the configuration of the system in the $\mathrm{H}$-zone depends on its history. This is like a hysteretic cycle in magnetism.

Let us comment again on the distinction between $\boldsymbol{L}$ and $\boldsymbol{\Omega}$. If we restrict ourselves to single-cluster axisymmetric solutions, as we do, we do not expect qualitative difference in the phase diagram according as we fix angular momentum or angular velocity. Indeed, there is a one-to-one correspondance between $\boldsymbol{\Omega}$ and $\boldsymbol{L}$. This is very different if we allow for nonaxisymmetric, or disconnected, solutions (such as double clusters, rings, ...) that are beyond the scope of the present paper. In that case, there is no one-to-one correspondance between $\mathbf{\Omega}$ and $\boldsymbol{L}$ and the choice of the control parameter is crucial. The thermal energy $K_{\text {th }}=E-W-\frac{1}{2} I \Omega^{2}$ is for a single central cluster at the same $E, \Omega$ much greater than for a double cluster with larger $I$ and $W$. Thus, in $(E, \Omega)$ ensemble, the central system has more entropy (thermal random motion) than the double cluster. Hence, the single cluster will overshadow the probability of the binary state (we thank the referee for this comment). On the other hand, a double cluster rotates more slowly than a single central cluster with same $L$. Therefore, in $(E, L)$ ensemble, a single cluster could avoid forming an equatorial cusp by splitting in two pieces. Depending on $L$ and $E$, the double cluster solution may have more entropy than the single one. It would be of interest to consider this problem in detail in the case of self-gravitating fermions. We do not know if "binary fermion balls" have been introduced in dark matter models. We can wonder whether the transition from single to double clusters (if any) occurs at or before the Keplerian limit. Again, we emphasize that in the case of self-gravitating fermions (similar to polytropes $n=3 / 2>0.808$ ), this transition would be discontinuous with respect to the spheroidal sequence.

\section{Caloric curves for high values of the degeneracy parameter (low cut-off/large system)}

We now consider the case of a high value of the degeneracy parameter $\mu=10^{5}>\mu_{\mathrm{MTP}}=2670$ (large system) so that the influence of the small-scale cut-off is weaker in average and the conditions required to set up a gravothermal catastrophe are fulfilled.

The caloric curve $T(E)$ is plotted in Fig. 6 for different values of angular velocity $\Omega$. It has the typical " $Z$-shape" structure (resembling a "dinosaur's neck") previously found ${ }^{2}$ for

${ }^{2}$ Of course, in a strict sense, the microcanonical caloric curve must be single valued everywhere because $S_{\text {micro }}(E)=\ln g(E)$ and $1 / T_{\text {micro }}(E)=\mathrm{d} S_{\text {micro }} / \mathrm{d} E$ (Gross 2001, 2003). However, the caloric curves that we represent are richer because they contain saddle points SP (unstable), local maxima LEM (metastable) and global maxima

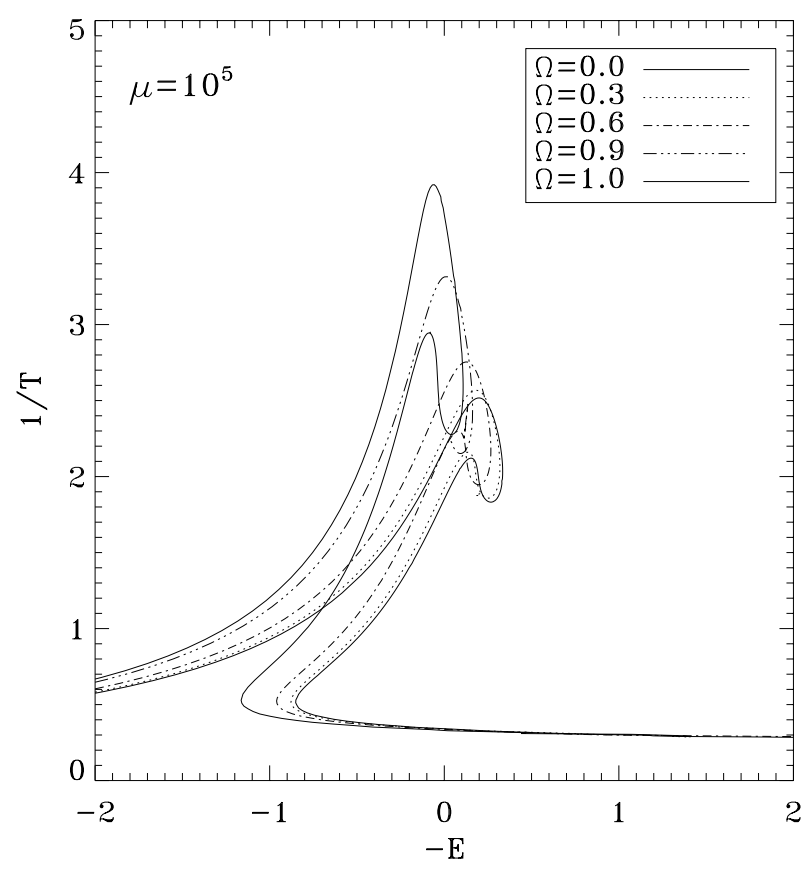

Fig. 6. The caloric curve of a rotating system with $\mu=10^{5}$. It has a $Z$-shape structure (dinosaur's neck) characteristic of self-gravitating systems with small cut-off $\left(\mu \geq \mu_{\mathrm{MTP}}=2670\right)$. The ground state $(T=$ $0, E=-138)$ is outside the range of represented energies.

non-rotating or slowly rotating systems (Chavanis \& Sommeria 1998; Chavanis 2002b,c). The upper branch corresponds to non-degenerate states (gaseous phase) and the lower branch corresponds to core-halo states (condensed phase). A first order microcanonical phase transition is expected to occur at $E_{\mathrm{t}}(\mu)$ since at this energy the two phases have the same entropy (Fig. 7). This gravitational phase transition is marked by a discontinuity of temperature and specific heats. If $E_{\mathrm{t}}>E_{\text {gas }}$ (where $E_{\text {gas }}$ is the first turning point of temperature), the specific heats passes from positive to negative values. If $E_{\mathrm{t}}<E_{\mathrm{gas}}$, the specific heats is always negative at the transition (the crossing point occurs for $\mu \simeq 1.10 \times 10^{4}$ ). However, this first order phase transition may not take place in practice because the gaseous states with energy $E_{\mathrm{c}}<E<E_{\mathrm{t}}$ are metastable (local entropy maxima) and long-lived (Katz \& Okamoto 2000; Chavanis \& Ispolatov 2002). The collapse (gravothermal catastrophe) will set in at, or near, the critical energy $E_{\mathrm{c}}$ (Antonov energy) at which the gaseous phase disapears. This corresponds

GEM (stable) of entropy. Had we represented only global entropy maxima, the curves $S(E)$ and $\beta(E)$ would be single-valued. They would describe the true statistical equilibrium states reached for $t \rightarrow$ $+\infty$. However, metastable states are of considerable importance in astrophysics because they correspond to the observed structures (e.g., globular clusters) for the timescales contemplated (as is well known, stellar systems are not in true statistical equilibrium states). Therefore, the physical caloric curves must take these metastable states into account and this gives rise to what we have nicknamed "dinosaur's necks". We recall that these metastable states appear only for sufficiently small cut-offs. For large cut-offs (see Fig. 1), there is only one global entropy maximum for each energy and the $\beta(E)$ curve is univalued. This is the situation considered by Votyakov et al. (2002) (compare their Fig. 7 to our Fig. 3). 


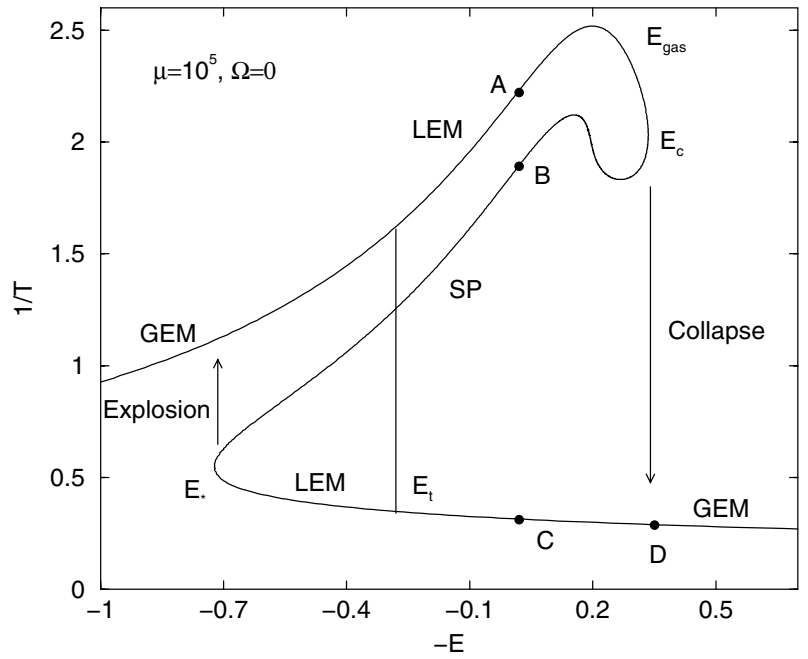

Fig. 7. Caloric curve of a self-gravitating system with $\mu=10^{5}$ (here $\Omega=0$ ). Between $E_{\mathrm{t}}$ and $E_{\mathrm{c}}$ (resp. $E_{*}$ ) the gaseous states (resp. condensed states) are metastable. A collapse occurs at $E_{\mathrm{c}}$ (gravothermal catastrophe) and an explosion occurs at $E_{*}$. By varying the energy of the system between $E_{*}$ and $E_{\mathrm{c}}$ (spinodal points), we can generate an hysteretic cycle in the microcanonical ensemble.

to a zeroth order microcanonical phase transition accompanied by a discontinuity of temperature and entropy. The collapse stops when the central region becomes degenerate and forms a dense nucleus. The resulting configuration has a "core-halo" structure. Contrary to the collapse in the canonical ensemble, the nucleus contains only a moderate fraction of mass. In the classical limit $\mu \rightarrow+\infty$, it reduces to a single binary (see Sect. 5). The rest of the mass is diluted in a hot envelop (with almost uniform density) that is held by the walls of the box. For an open system, the halo would be dispersed at infinity so that only the degenerate nucleus would remain. This behaviour, with the expulsion of an envelope, is more consistent with the process of white dwarf formation than a complete collapse of the system at fixed $T$. This is an astrophysical evidence that the microcanonical ensemble is more appropriate than the canonical one. The condensed states with energy $E_{\mathrm{t}}<E<E_{*}$ are also metastable and long-lived. An "explosion", reversed to the gravothermal catastrophe, will occur above $E_{*}$ if the system is initially prepared in the condensed phase (see Fig. 7). By varying the energy between $E_{*}$ and $E_{\mathrm{c}}$ (microcanonical spinodal points) we can generate an hysteretic cycle in the microcanonical ensemble (Chavanis 2003b).

The phase diagram describing the microcanonical phase transition is represented in Fig. 8. It can be compared with the phase diagram describing the canonical phase transition for $\mu=100$ (see Fig. 5), the role of energy $E$ and temperature $T$ being reversed. In particular, there exists a $H$-zone in which the structure of the system (gas or condenstate) depends on its history. For smaller energies $\left(E<E_{\mathrm{c}}\right)$, the system is in a mixed phase with negative specific heats. For even lower energies $E_{\text {cond }} \sim-117$ (not represented on the diagram), we leave the region of negative specific heats and enter the pure condensed phase as in Fig. 4. More details on these phase transitions can be found in Chavanis (2002b). It is shown in

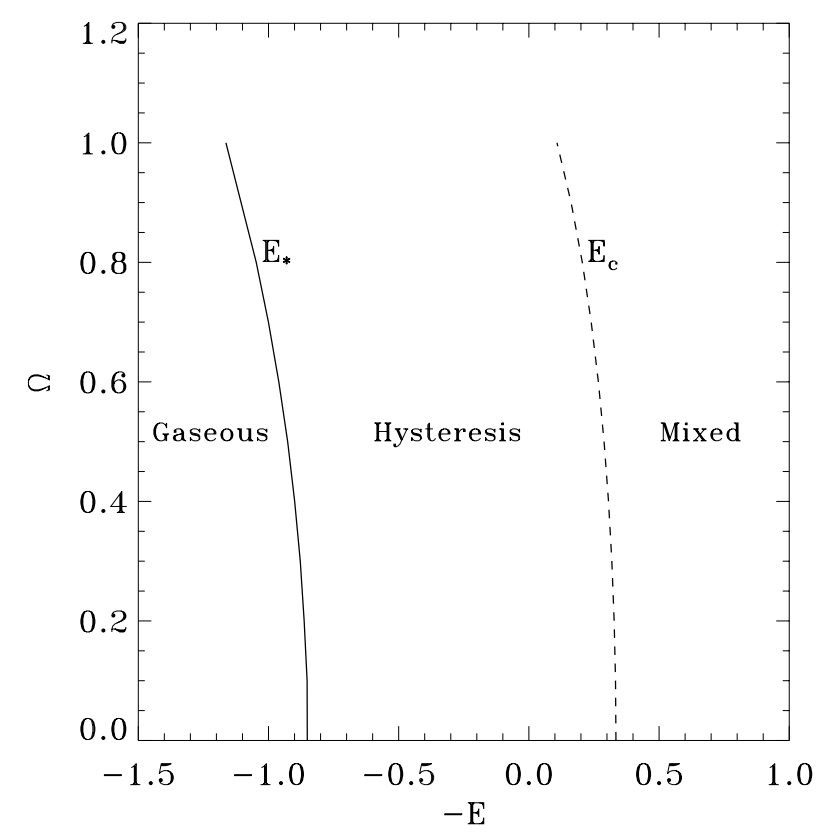

Fig. 8. Microcanonical phase diagram for $\mu=10^{5}$. The $H$-zone corresponds to an hysteretic zone where the actual phase depends on the history of the system.

particular that the microcanonical first order phase transition disapears for $\mu<\mu_{\mathrm{MTP}}=2670$.

\section{Gravothermal catastrophe for classical self-gravitating particles (no cut-off)}

We now consider the limit $\mu=+\infty$ (or $h=0$ ) corresponding to classical particles. In that limit, the particles do not feel any kind of exclusion or small-scale cut-off. This is the situation relevant for globular clusters for which the size of the stars does not matter.

In the non degenerate limit, the caloric curve has a classical spiral shape (Fig. 9). We recall that, in the absence of smallscale cut-off, the equilibrium states are only metastable as they correspond to local maxima of the thermodynamical potential (entropy or free energy). These metastable states play however an important role as they correspond to the observed structures (see discussion in Chavanis 2003a). In the microcanonical ensemble, the gravothermal catastrophe sets in at the Antonov energy $E_{\mathrm{c}}$. The microcanonical phase diagram in $(E, \Omega)$ plane is drawn in Fig. 10 and shows in particular the evolution of the Antonov critical energy $E_{\mathrm{c}}(\Omega)$ with the rotation. The critical energy $E_{\mathrm{c}}(\Omega)$ increases with rotation so that gravitational collapse occurs sooner than in the non-rotating case. This confirms the results of the perturbative approach (Chavanis 2002c). Qualitatively, the critical energy increases due to the rotational term $\frac{1}{2} I \Omega^{2}>0$ in the expression of $E$ (see Sect. 2.5). The result of the collapse is to form a binary star with large binding energy. Since total energy is conserved, the gravitational energy released by the binary is redistributed in a dilute halo in the form of thermal energy. This evolution is natural in the microcanonical ensemble since it can produce very high (ideally diverging) values of entropy due to the divergence of the 


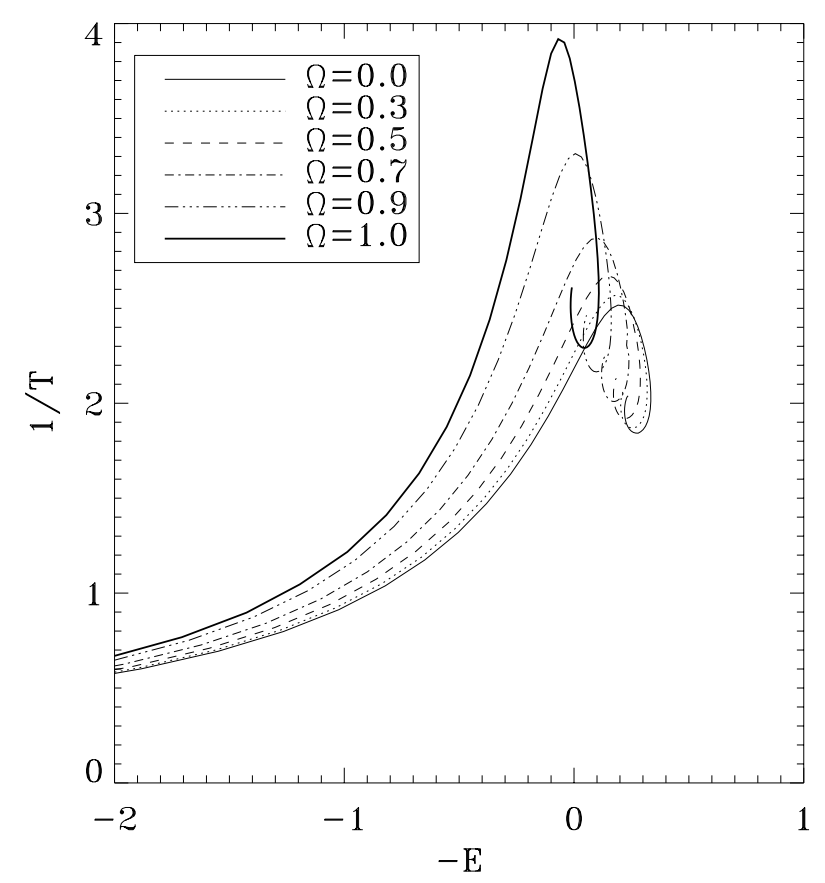

Fig. 9. Caloric curves corresponding to classical point masses $(\mu=$ $+\infty$ ) for different values of angular velocity. Below $E_{\mathrm{c}}$, the gravothermal catastrophe leads to the formation of a binary star (microcanonical ensemble). Below $T_{\mathrm{c}}$, the isothermal collapse generates a Dirac peak (canonical ensemble).

temperature. It is also vindicated by numerical simulations of the orbit averaged Fokker-Planck equation (Cohn 1980) or fluid equations (Lynden-Bell \& Eggleton 1980). These simulations show that the collapse of globular clusters is self-similar and leads to a finite time singularity with a density profile $\rho \sim r^{-\alpha}$ with $\alpha \simeq 2.2$. The mass contained in the core goes to zero (indicating that the collapse is halted by the formation of binaries with mass $2 m \ll M$ ) while the central temperature raises to $+\infty$ since $\alpha>2$. Binary-like structures are also found in the microcanonical model of self-gravitating Brownian particles (Sire \& Chavanis 2003).

In the canonical ensemble, the collapse sets in at the critical temperature $T_{\mathrm{c}}$. The evolution of this critical temperature with the angular velocity is represented in Fig. 10. It is found that $T_{\mathrm{c}}(\Omega)$ decreases with $\Omega$ so that gravitational collapse is delayed in the canonical ensemble. This corroborates the results found with the perturbative approach (Chavanis 2002c). The result of the collapse is to form a Dirac peak, i.e. a state where all the mass is concentrated at the center ("black hole"). This evolution is natural in the canonical ensemble since it can produce very high (in fact diverging) values of free energy $J=S-\beta E$ due to the divergence of the potential energy. This isothermal collapse can be followed dynamically by solving the Euler-Jeans equations for a gaseous system with an equation of state $p=\rho T$ where $T$ is fixed (Penston 1969). It can also be studied analytically in the canonical model of self-gravitating Brownian particles (Chavanis et al. 2002). In this model, there is first a finite time singularity leading to a $r^{-2}$ density profile. Then, the collapse continues after the singularity is formed and the Dirac peak appears in the post-collapse regime due to the accretion of mass (Sire \& Chavanis 2002, 2003). We expect this two-stage behaviour to persist in the case of more relevant dynamical models. This may be an important point for numerical simulations in astrophysics and cosmology because if we only consider the first regime we conclude that the core contains no mass (while its density is high and scales as $r^{-2}$ ). However, in a post-collapse regime, a Dirac peak is formed which contains a macroscopic mass at a single point. We can wonder whether this mechanism (apparently not reported before) is related to the existence of black holes at the center of galaxies. In reality, small-scale cut-offs must be introduced (e.g., quantum degeneracy) and the black hole singularity is replaced by a "fermion ball" with a high mass. Note that in the microcanonical ensemble, there is no black hole singularity for classical point masses (the Dirac peak is replaced by a binary). Interestingly, for self-gravitating fermions, the condensate (fermion ball) contains a moderate but macroscopic fraction of mass (Chavanis \& Sommeria 1998). This may explain the formation of massive objects at the center of galaxies in a microcanonical framework. The microcanonical description is the most relevant as it accounts for the transfers of energy between the core and the halo contrary to isothermal models.

The divergence of entropy in the microcanonical ensemble due to the formation of a binary embedded in a hot halo is implicit in the work of Antonov (1962). It is also clear from the arguments given by Padmanabhan (1990) to show that the density of state diverges as we approach two particles to each other provided that thermal energy can be redistributed in a halo (this demands $N \geq 3$ ). The divergence of free energy in the canonical ensemble by forming a Dirac peak has been shown rigorously by Kiessling (1989) and heuristically by Chavanis (2002e). The inequivalence of statistical ensembles regarding the formation of binaries or Dirac peaks is further discussed in Appendices A and B of Sire \& Chavanis (2002). In this approach, a family of distributions functions is constructed which provokes the divergence of entropy or free energy when a physical parameter is varied. These results can also be obtained by considering the small cut-off limit $\mu \rightarrow+\infty$ in the analytical model of phase transitions for self-gravitating systems proposed by Chavanis (2002b).

\section{Phase diagram of non-rotating systems}

In previous sections, we have presented the equilibrium phase diagram of self-gravitating fermions as a function of angular velocity $\Omega$ for a fixed value of degeneracy parameter $\mu$. We shall now discuss the equilibrium phase diagram of selfgravitating fermions (or hard sphere systems) as a function of $\mu$ for $\Omega=0$. These phase diagrams have not been published before.

The deformation of the caloric curve $T(E)$ as a function of the degeneracy parameter $\mu$ is described in detail by Chavanis (2002b). This work completes earlier studies on phase transitions in self-gravitating systems by showing the small cutoff limit, characterized by an unwound spiral ("dinosaur's neck"), leading to a first order microcanonical phase transition (earlier works essentially described the canonical first order phase transition). Typical curves illustrating first order 

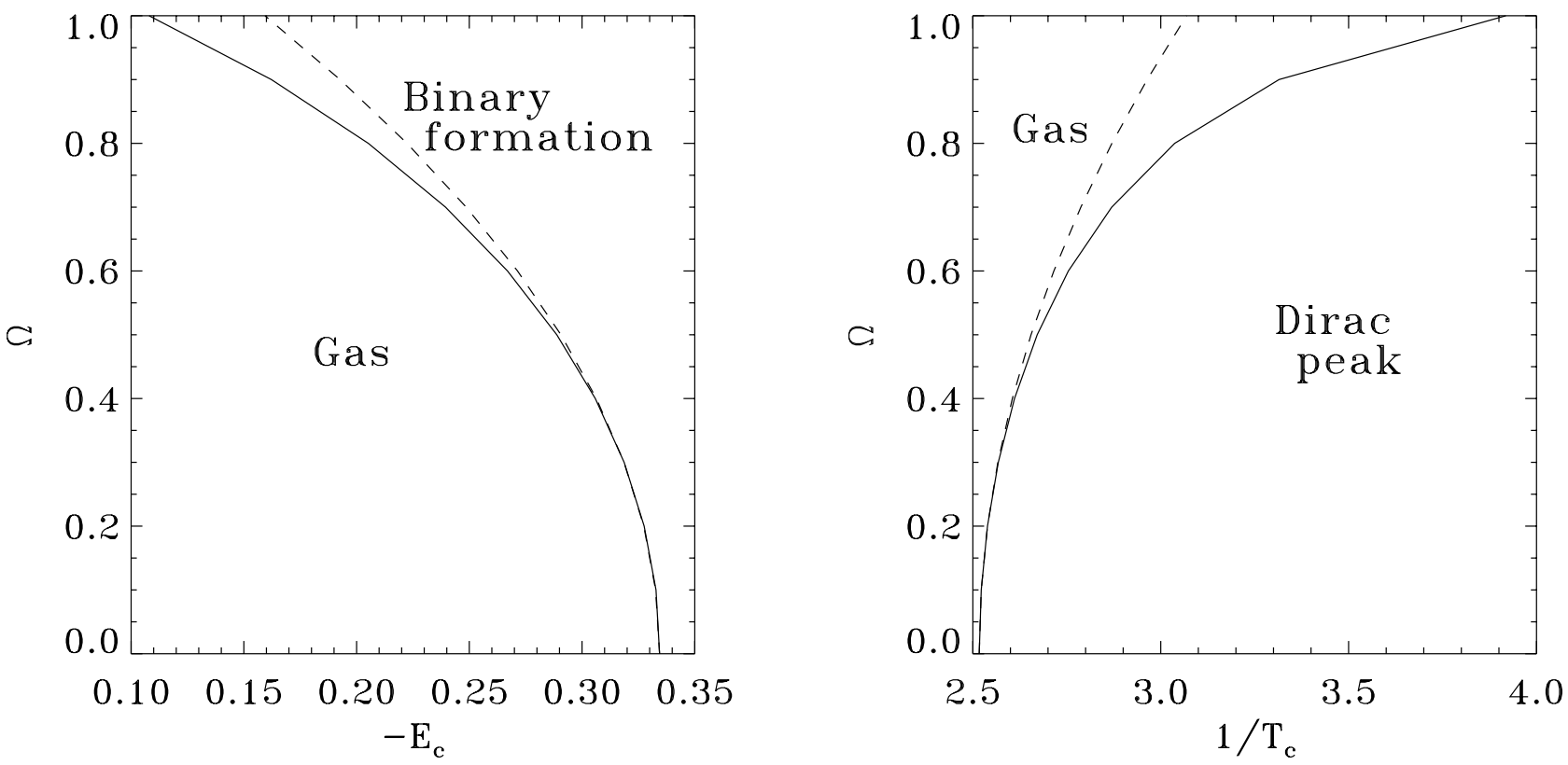

Fig. 10. Dependence of the Antonov critical energy and critical temperature with rotation. For high energies or high temperatures, the system is in a gaseous (metastable) phase. Below a critical energy or temperature, it enters in the collapsed phase. In the microcanonical ensemble (fixed $E$ ), the collapse leads to a single binary (or a small group of stars) surrounded by a hot dilute halo. In the canonical ensemble (fixed $T$ ), the collapse leads to a Dirac peak containing all the mass ("black hole"). This Dirac peak forms in the post-collapse regime of the dynamical evolution. For small rotations, the critical energy and critical temperature behave as $E_{\mathrm{c}}(\Omega)=E_{\mathrm{c}}(0)+0.175 \Omega^{2}$ and $1 / T_{\mathrm{c}}(\Omega)=1 / T_{\mathrm{c}}(0)+0.552 \Omega^{2}$ (dotted lines). This scaling is valid typically for $\Omega \leq 0.5$.

canonical and first order microcanonical phase transitions are shown in Figs. 1 and 7. The equilibrium phase diagram of selfgravitating fermions can be directly deduced from these results by identifying characteristic energies and temperatures. In the canonical ensemble, we note $T_{\mathrm{t}}$ the temperature of transition (determined by the equality of the free energies of the two phases), $T_{\mathrm{c}}$ the end point of the metastable gaseous phase (first turning point of temperature) and $T_{*}$ the end point of the metastable condensed phase (last turning point of temperature). The temperature of transition $T_{\mathrm{t}}$ is determined by a horizontal Maxwell construction (Chavanis 2003b). The canonical phase diagram is shown in Fig. 11. It shows in particular the canonical critical point $\mu_{\text {СTP }}=83$ at which the canonical first order phase transition disappears and is replaced by a localized second order phase transition.

In the microcanonical ensemble, we note $E_{\mathrm{t}}$ the energy of transition (determined by the equality of the entropy of the two phases), $E_{\mathrm{c}}$ (Antonov energy) the end point of the metastable gaseous phase (first turning point of energy) and $E_{*}$ the end point of the metastable condensed phase (last turning point of energy). The energy of transition $E_{\mathrm{t}}$ is determined by a less standard vertical Maxwell construction (Chavanis 2003b). We also denote by $E_{\text {gas }}$ the energy at which we enter in the mixed phase with negative specific heat (first turning point of temperature) and $E_{\text {cond }}$ the energy at which we leave the mixed phase and enter the condensed phase (last turning point of temperature). We also introduce the minimum energy $E_{\text {min }}$ (ground state). The microcanonical phase diagram is shown in Fig. 12. It shows in particular the microcanonical critical point $\mu_{\mathrm{MTP}}=$ 2670 at which the microcanonical first order phase transition disappears. The structure of the equilibrium phase diagrams

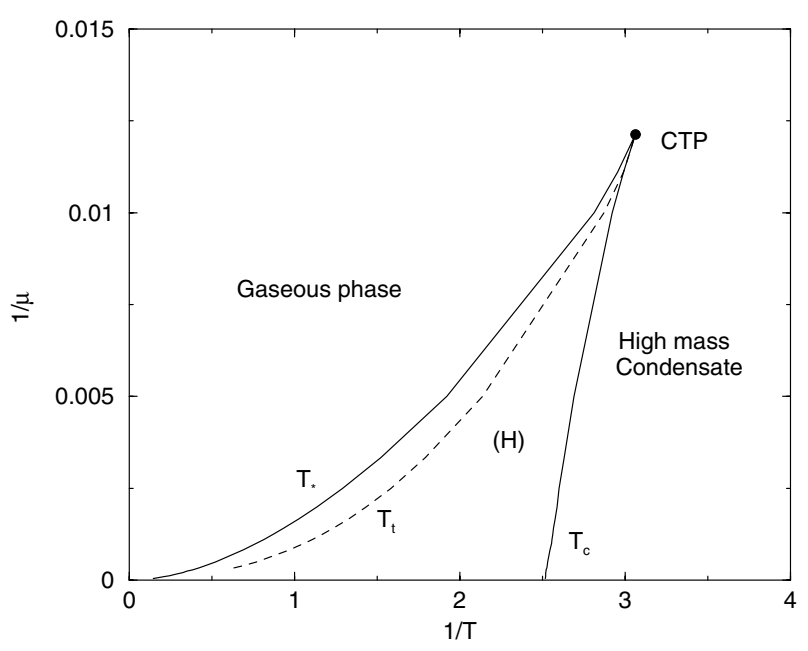

Fig. 11. Canonical phase diagram in $(T, \mu)$ plane for $\Omega=0$. The $H$-zone corresponds to an hysteretic zone where the actual phase depends on the history of the system.

can be easily understood in the light of the preceding discussion (see Chavanis 2002b for more details). We note that the microcanonical phase diagram is more complex than the canonical one due to the existence of a negative specific heat region. We recall that canonical stability (maximum of $J$ at fixed $M, \beta$ ) is a sufficient, albeit not necessary, condition of microcanonical stability (maximum of $S$ at fixed $M, E$ ). Since canonical equilibria are always realized as microcanonical equilibria, they constitute a sub-domain of the microcanonical phase diagram. Hence, the microcanonical ensemble is richer than the canonical one, as is well-known. 


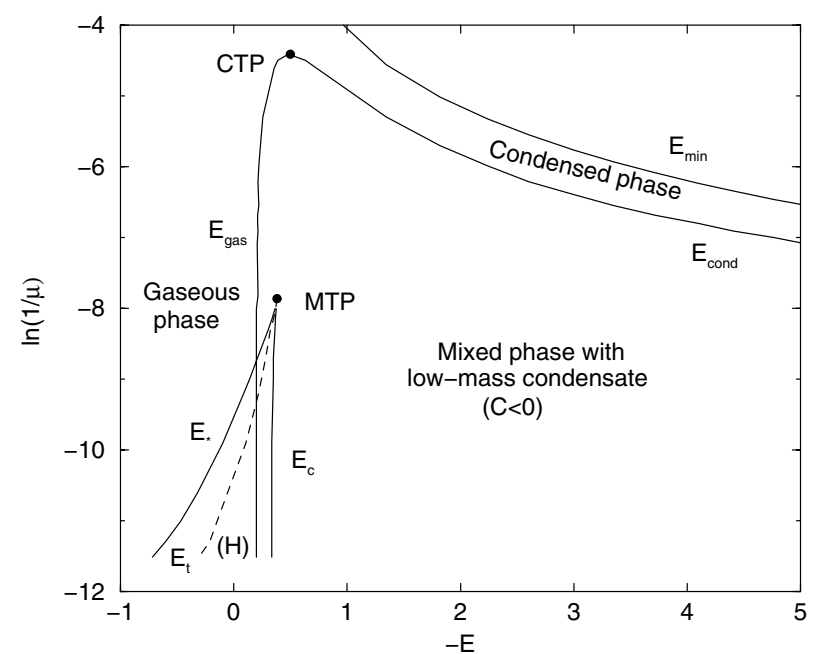

Fig. 12. Microcanonical phase diagram in $(E, \mu)$ plane for $\Omega=0$. The phase diagram in MCE is more complex than in CE due to the existence of the negative specific heat region that is forbidden in $\mathrm{CE}$.

\section{Conclusion}

We have calculated single-cluster axisymmetric statistical equilibrium states of self-gravitating fermions for arbitrary values of rotation and temperature (or energy). The problems associated with the divergence of the gravitational potential as $r \rightarrow 0$ have been avoided by considering quantum effects. This smallscale regularization is a physical one because the Pauli exclusion principle is a fundamental concept and fermionic matter is relevant in astrophysics. Isothermal and polytropic configurations (with index $n=3 / 2$ ) are obtained as particular limits of our model $(T \rightarrow+\infty$ and $T=0)$. On the other hand, depending on the importance of rotation, we have obtained different types of structures such as fermion balls, fermion spheroids and cuspy structures. In the mixed phase, these condensed objects are surrounded by a diffuse isothermal halo. Clearly, the main drawback of our study is the necessity to enclose the system within an artificial box so as to prevent its complete evaporation (which is the true statistical equilibrium state for self-gravitating systems). A future extension of our study is to consider more realistic truncated models in order to avoid the artifice of a material box and take into account incomplete relaxation or tidal effects. We note, in passing, that the distribution function $f=f\left(\epsilon_{\mathrm{J}}\right)$ predicted by statistical mechanics cannot account for the triaxial structure of elliptical galaxies since no bifurcation from axisymmetric (spheroidal) to non-axisymmetric (ellipsoidal) structures are expected for uniformly rotating compressible bodies. The distribution function must depend on isolated integrals different from the single Jacobi integral (Contopoulos 1960). This is clearly a manifestation of incomplete relaxation but the construction of such isolated integrals is a highly complicated task and demands a very delicate analysis of the process of formation of elliptical galaxies.

We emphasize that the nature of the small-scale cut-off is of considerable importance for rotating objects, regarding the structure of the condensed phase, because it can generate or inhibit non-axisymmetric instabilities. For example, the ordinary Fermi-Dirac distribution used in this paper and the model of Votyakov et al. (2002) have the same high temperature limit (an isothermal gas with $p=\rho T$ ) but a different low temperature limit. The Fermi gas at $T=0$ corresponds to the equation of state $p=K \rho^{5 / 3}$ (polytropic core). The equation of state corresponding to a Fermi-Dirac distribution in configuration space $\rho=\rho_{\max } /\left[1+\lambda \exp \left(\beta \Phi_{\text {eff }}\right)\right]$ is $p=T \ln \left(1-\rho / \rho_{\max }\right)$, as can be obtained from the condition of hydrostatic equilibrium $^{3}$. For $T=0, \rho$ is a step function with $\rho \rightarrow \rho_{\max }$ for $r<R_{*}$ (solid core) and $\rho=0$ otherwise. In the former case (fermions), there is no bifurcation to non-axisymmetric structures at high rotations in continuity with the spheroidal sequence. Instead, the system develops an equatorial cusp (the possibility of discontinuous bifurcations to non-axisymmetric structures will be considered in a future work). This cusp does not exist for homogeneous bodies (or hard sphere systems) and non-axisymmetric bifurcations occur continuously at high rotations. It is surprising that the equation of state corresponding to the model of Votyakov et al. (2002) does not coincide with the Van der Waals equation of state, as in the work of Aronson \& Hansen (1972), since these authors treat their system as a hard sphere gas ${ }^{4}$. It is also surprising that they do not obtain a richer variety of structures in the low temperature/energy regime (pear shaped, dumbbell, ...) since rotating homogeneous bodies possess a rich bifurcation diagram as investigated since the 19th century (Chandrasekhar 1969). These remarks show that the small-scale regularization must be given precise consideration depending on the situation contemplated.

The self-gravitating Fermi gas has several astrophysical applications: (i) Lynden-Bell's type of degeneracy may be relevant for galactic nuclei and dark matter (Lynden-Bell 1967; Kull et al. 1996; Chavanis \& Sommeria 1998; Stiavelli 1998; Leeuwin \& Athanassoula 2000). (ii) Pauli's exclusion principle is relevant for white dwarfs and neutron stars (Chandrasekhar 1942; Hertel \& Thirring 1971). In that context, the Fermi gas at non-zero temperature provides a simple theoretical model describing a phase transition from a "gaseous star" (for $T>T_{\mathrm{c}}$ )

\footnotetext{
${ }^{3}$ A Fermi-Dirac distribution in configuration space also arises in the statistical mechanics of two-dimensional vortices (see Chavanis 2002a for a review on the analogy between stellar systems and 2D vortices). In that respect, it may be relevant to mention the work of Chen \& Cross (1996) who found "double-vortex" equilibrium solutions in a circular domain when the conservation of angular momentum is accounted for. This can be viewed as the $2 \mathrm{D}$ hydrodynamical version of the "double-star" structure found by Votyakov et al. (2002). Note that the presence of a confining box is crucial to maintain the double-vortex solution. Similarly, for self-gravitating systems at nonzero temperature, an artificial box is needed to maintain the doublestar structure.

${ }^{4}$ In fact, Votyakov et al. (2002) prevent overlapping of particles in physical space, as in a lattice gas model. This is a simple way to limit the local density $\rho(\boldsymbol{r})$ of the self-gravitating gas. Hence, their model is expected to represent stars at densities up to the ignition of their hydrogen burning, when further gravitational collapse is halted for a while (Gross 2003). In this context, it may be relevant to consider a large small-scale cut-off, as they do. Note that their model does not correspond to the Lynden-Bell (1967) statistics, which is a Fermi-Dirac distribution in phase space appropriate to collisionless stellar systems (e.g., Chavanis 2002d).
} 
to a white dwarf or a neutron star (for $T<T_{\mathrm{c}}$ ). To obtain a better description of these objects, special relativity must be taken into account for massive white dwarf stars (Chandrasekhar 1931) and general relativity is required in the case of neutron stars (Oppenheimer \& Volkoff 1939). (iii) It has been proposed recently that dark matter could be made of a collisionless gas of massive neutrinos. Therefore, the relevant distribution function is again the Fermi-Dirac distribution (see, e.g., Chavanis 2002f). The degeneracy is due either to the Liouville theorem (in the context of violent relaxation) or to Pauli's exclusion principle (if quantum effects are relevant). By cooling below a critical temperature, this neutrino gas is expected to undergo a phase transition to a compact object called a "fermion ball". It has been proposed that fermion balls could provide an alternative to black holes at the center of galaxies (Bilic \& Viollier 1997). (iv) Incidentally, our study can also be connected to models of rotating stars (e.g., Roxburgh et al. 1965). Low mass stars $\left(M \lessgtr 1.5 M_{\odot}\right)$ of the main sequence have a radiative core and a convective envelope. Massive stars $\left(M>1.8 M_{\odot}\right)$ have the opposite configuration. Now, the convective region corresponds to a polytrope of index $n=3 / 2$. Coincidentally, this case is precisely covered by our study.

On the other hand, hard spheres models have less direct applications in astrophysics because the inter-particle distance is always considerably larger than their size. Indeed, globular clusters can be either in dilute metastable equilibrium states (Michie 1963; King 1966) or in a collapsing phase ending up with the formation of binaries (Hénon 1961). These binaries can release sufficient energy to drive a re-expansion of the system (Inagaki \& Lynden-Bell 1983; Bettwieser \& Sugimoto 1984) so that complete collapse is prevented in practice. In each case, the size of the stars does not matter. The size of the atoms in stars does not matter neither because radiative transfers keep the star in a gaseous phase far from condensation. When a star collapses (because it has burned its nuclear fuel), gravity is balanced by quantum pressure not by the pressure of a hard sphere gas. In that respect, it may be useful to recall that the hard sphere model of Aronson \& Hansen (1972) was presented as a crude model for neutron stars where the hard spheres were introduced to mimick quantum degeneracy. The hard-sphere model can however find physical applications in the context of planet formation resulting from the gravitational collapse of dust particles in the solar nebula (Chavanis 2000). It has also been used by Stahl et al. (1994) to describe the formation of stars or planetoids (considered as the solid phase) resulting from the condensation of a molecular cloud (considered as the gaseous phase). In this context, the explosion, reverse to the collapse, may be related to the supernova phenomenon.

Finally, our study has a direct relevance in terms of fundamental statistical mechanics. Indeed, the different types of phase transitions arising in the self-gravitating Fermi gas (Chavanis 2002b) display a very rich structure: inequivalence of statistical ensembles, negative specific heats, convex intruder in the entropy-energy curve, canonical and microcanonical first order phase transitions, second order phase transition, zeroth order phase transition, canonical and microcanonical critical points, metastable states, hysteretic cycles, ... Such properties are probably common to other systems with long-range interactions. In fact, the self-gravitating Fermi gas forms a generic example of systems displaying codimensions 0 and 1 singularities in the classification of phase transitions (for longrange interactions) recently proposed by Bouchet \& Barré (2003). Therefore, the detailed study of phase transitions in the self-gravitating Fermi gas at non-zero temperature is of interest both for statistical mechanics and astrophysics. This point is important because the statistical mechanics of self-gravitating systems was considered until recently, as a curiosity, not to say a fallacy, by statistical mechanicians.

An extension of our study would be to compute more complex configurations of rapidly rotating self-gravitating fermions and $n=3 / 2$ polytropes (double clusters, rings and core-ring sequences) in both microcanonical (fixed $E, M$ and $\boldsymbol{L}$ ) and canonical (fixed $T, M$ and $\mathbf{\Omega}$ ) ensembles. These solutions are expected to bifurcate discontinuously from the spheroidal sequence in contrast to hard sphere systems and homogeneous bodies $(n=0)$. It would be important (but difficult) to investigate the stability of these various configurations. For rigid bodies $(n<0.808$ polytropes and hard sphere systems at low temperature), the spheroidal sequence becomes unstable (saddle points of $S$ or $J$ ) above a certain rotation at which the branch of non-axisymmetric structures appears. For high rotations, double clusters are global maxima of $S$ or $J$ and the ring sequence is always unstable (saddle points of $S$ or $J$ ). For compressible bodies $(n>0.808$ polytropes and fermionic matter at low temperature), the spheroidal sequence is expected to remain stable (maxima of $S$ or $J$ ) until the Keplerian limit since no continuous bifurcations exist. However, it is possible that, for high rotations, the single cluster structures computed in this paper are only metastable (local maxima of $S$ or $J$ ) and that the true equilibrium states (global maxima of $S$ or $J$ ) are double clusters or core-ring configurations. In case when several stable solutions exist for the same values of $E, \boldsymbol{L}$ ( or $\beta, \boldsymbol{\Omega}$ ), it may be important to compare their entropy and free energy. However, the selection of the physical equilibrium state will depend more on a notion of basin of attraction (see, e.g., Chavanis et al. 2002 in a related context) than whether it is a global or a local maximum of $S$ or $J$. Thus, a dynamical study is required. We hope to come to these problems in future communications. The present paper is only a first step in that direction. To be more realistic, the box could be avoided by using the distribution function (15) which provides a more physical confinement of the system. We also emphasize that in a large domain, the conservation of angular momentum can be satisfied by ejecting a weak amount of mass at large distances, for example in the form of a Keplerian disk. This is the typical structure of a protoplanetary nebula. Indeed, during the formation of a (single) star by gravitational collapse of a molecular cloud, about $99 \%$ of the intial angular momentum is spread in a protoplanetary disk while $99 \%$ of the mass remains in the star itself whose internal structure is hardly affected by rotation.

Acknowledgements. We thank the referee, D. H. E. Gross, for a critical reading of the manuscript. Although we share a different point of view concerning the importance of metastable states in astrophysics, his comments were very useful (a criticism raised in his referee report is available on cond-mat/0307535). One of us (PHC) is also 
grateful to O. Fliegans, I. Ispolatov, J. Katz and V. Laliena for valuable discussions.

\section{References}

Antonov, V. A. 1962, Vest. Leningr. Gos. Univ., 7, 135

Aronson, E. B., \& Hansen, C. J. 1972, ApJ, 177, 145

Bettwieser, E., \& Sugimoto, D. 1984, MNRAS, 208, 493

Bilic, N., \& Viollier, R. D. 1997, Phys. Lett. B, 408, 75

Binney, J., \& Tremaine, S. 1987, Galactic Dynamics (Princeton Series in Astrophysics)

Bonnor, W. B. 1956, MNRAS, 116, 351

Boss, A. P. 1986, Icarus, 66, 330

Boss, A. P. 1988, Comm. Astrophys., 12, 169

Bouchet, F., \& Barré, J. 2003, J. Stat. Phys., submitted

Brands, H., Chavanis, P. H., Pasmanter, R., \& Sommeria, J. 1999, Phys. Fluids, 11, 3465

Chandrasekhar, S. 1931, ApJ, 74, 81

Chandrasekhar, S. 1933, MNRAS, 93, 390

Chandrasekhar, S. 1942, An Introduction to the Theory of Stellar Structure (Dover)

Chandrasekhar, S. 1969, Ellipsoidal figures of equilibrium (New Haven: Yale University Press)

Chavanis, P. H. 1998, MNRAS, 300, 981

Chavanis, P. H. 2000, A\&A, 356, 1089

Chavanis, P. H. 2002a, in Dynamics and thermodynamics of systems with long range interactions, ed. T. Dauxois, S. Ruffo, E. Arimondo, \& M. Wilkens, Lecture Notes in Physics (Springer) [cond-mat/0212223]

Chavanis, P. H. 2002b, PRE, 65, 056123

Chavanis, P. H. 2002c, A\&A, 396, 315

Chavanis, P. H. 2002d, in Proc. of the Conf. on Multiscale Problems in Science and Technology, ed. N. Antonic, C. J. van Duijn, W. Jäger, \& A. Mikelic (Berlin: Springer) [astro-ph/0212205]

Chavanis, P. H. 2002e, A\&A, 381, 340

Chavanis, P. H. 2002f, in Proc. Fourth International Heidelberg Conf. Dark Matter in Astro and Particle Physics, ed. H. V. Klapdor-Kleingrothaus (New-York: Springer) [astro-ph/0205426]

Chavanis, P. H. 2003a, A\&A, 401, 15

Chavanis, P. H. 2003b, in Proc. TH-2002 conf. Theoretical Physics, ed. J. Zinn-Justin, D. Iagolnitzer, \& V. Rivasseau (CD-rom)

Chavanis, P. H. 2003c, PRE, 68, 036108

Chavanis, P. H., \& Ispolatov, I. 2002, PRE, 66, 036109

Chavanis, P. H., Ribot, M., Rosier, C., \& Sire, C. 2003, in preparation

Chavanis, P. H., Rosier, C., \& Sire, C. 2002, PRE, 66, 036105

Chavanis, P. H., \& Sommeria, J. 1998, MNRAS, 296, 569

Chen, P., \& Cross, M. C. 1996, PRE, 53, R3032
Cohn, H. 1980, ApJ, 242, 765

Contopoulos, G. 1960, Z. Astrophys., 49, 273

de Vega, H. J., \& Sanchez, N. 2002, Nucl. Phys. B, 625, 409

Emden, R. 1907, Gaskugeln (Leipzig: Teubner Verlag)

Fliegans, O., \& Gross, D. H. 2002, PRE, 65, 6143

Follana, E., \& Laliena, V. 2000, PRE, 61, 6270

Fowler, R. H. 1926, MNRAS, 87, 114

Gross, D. H. E. 2001, Microcanonical thermodynamics: Phase transitions in Small systems (Singapore: World Scientific)

Gross, D. H. E. 2003 [cond-mat/0307535]

Hachisu, I., \& Eriguchi, Y. 1984, PASJ, 36, 239

Hénon, M. 1961, Ann. Astrophys. 5, 369

Hertel, P., \& Thirring, W. 1971, in Quanten und Felder, ed. H. P. Dürr (Vieweg, Braunschweig)

Hjorth, J., \& Madsen, J. 1993, MNRAS, 265, 237

Inagaki, S., \& Lynden-Bell, D. 1983, MNRAS, 205, 913

Ipser, J. R., \& Horwitz, G. 1979, ApJ, 232, 863

James, R. A. 1964, ApJ, 140, 552

Kandrup, H. 1981, ApJ, 244, 316

Katz, J. 1978, MNRAS, 183, 765

Katz, J., \& Okamoto, I. 2000, MNRAS, 371, 163

Kiessling, M. 1989, J. Stat. Phys. 55, 203

King, I. R. 1966, ApJ, 71, 64

Kull, A., Treumann, R. A., \& Böringer, H. 1996, ApJ, 466, L1

Lagoute, C., \& Longaretti, P. Y. 1996, A\&A, 308, 441

Laliena, V. 1999, PRE, 59, 4786

Laliena, V. 2003, Nucl. Phys. B, 668, 403

Leeuwin, F., \& Athanassoula, E. 2000, MNRAS, 417, 79

Lynden-Bell, D. 1967, MNRAS, 136, 101

Lynden-Bell, D., \& Eggleton, P. P. 1980, MNRAS, 191, 483

Lynden-Bell, D., \& Lynden-Bell, R. M. 1977, MNRAS, 181, 405

Lynden-Bell, D., \& Wood, R. 1968, MNRAS, 138, 495

Michie, R. W. 1963, MNRAS, 125, 127

Milne, E. A. 1923, MNRAS, 83, 118

Ogorodnikov, K. F. 1965, Dynamics of stellar systems (Pergamon)

Oppenheimer, J. R., \& Volkoff, G. M. 1939, Phys. Rev., 55, 374

Padmanabhan, T. 1990, Phys. Rep., 188, 285

Penston, M. V. 1969, MNRAS, 144, 425

Roxburgh, I. W., Griffith, J. S., \& Sweet, P. A. 1964, Z. Astrophys., 61,203

Sire, C., \& Chavanis, P. H. 2002, PRE 66, 046133

Sire, C., \& Chavanis, P. H. 2003 [cond-mat/0306406]

Stahl, B., Kiessling, M. K. H., \& Schindler, K. 1994, Planet. Space Sci. 43, 271

Stiavelli, M. 1998, ApJ, 495, L91

Thirring, W. 1970, Z. Phys., 235, 339

Tremaine, S., Hénon, M., \& Lynden-Bell, D. 1987, MNRAS, 227, 543

Votyakov, E. V., De Martino, A., \& Gross, D. H. E. 2002, Eur. Phys. J. B, 29, 593 